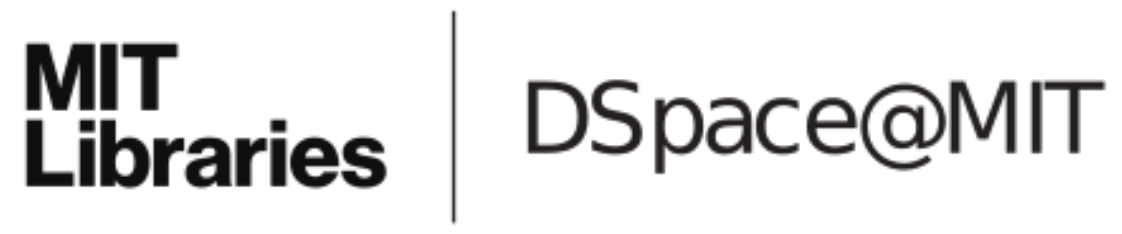

\author{
MIT Open Access Articles
}

Julia: A Fresh Approach to Numerical Computing

The MIT Faculty has made this article openly available. Please share how this access benefits you. Your story matters.

Citation: Bezanson, Jeff; Edelman, Alan; Karpinski, Stefan and Shah, Viral B. “Julia: A Fresh Approach to Numerical Computing." SIAM Review 59, no. 1 (January 2017): 65-98 () 2017 Society for Industrial and Applied Mathematics

As Published: http://dx.doi.org/10.1137/141000671

Publisher: Society for Industrial and Applied Mathematics

Persistent URL: http://hdl.handle.net/1721.1/110125

Version: Final published version: final published article, as it appeared in a journal, conference proceedings, or other formally published context

Terms of Use: Article is made available in accordance with the publisher's policy and may be subject to US copyright law. Please refer to the publisher's site for terms of use. 


\title{
Julia: A Fresh Approach to Numerical Computing*
}

\author{
Jeff Bezanson ${ }^{\dagger}$ \\ Alan Edelman \\ Stefan Karpinski ${ }^{\S}$ \\ Viral B. Shah ${ }^{\dagger}$
}

\begin{abstract}
Bridging cultures that have often been distant, Julia combines expertise from the diverse fields of computer science and computational science to create a new approach to numerical computing. Julia is designed to be easy and fast and questions notions generally held to be "laws of nature" by practitioners of numerical computing:

1. High-level dynamic programs have to be slow.

2. One must prototype in one language and then rewrite in another language for speed or deployment.

3. There are parts of a system appropriate for the programmer, and other parts that are best left untouched as they have been built by the experts.

We introduce the Julia programming language and its design - a dance between specialization and abstraction. Specialization allows for custom treatment. Multiple dispatch, a technique from computer science, picks the right algorithm for the right circumstance. Abstraction, which is what good computation is really about, recognizes what remains the same after differences are stripped away. Abstractions in mathematics are captured as code through another technique from computer science, generic programming.

Julia shows that one can achieve machine performance without sacrificing human convenience.
\end{abstract}

Key words. Julia, numerical, scientific computing, parallel

AMS subject classifications. $68 \mathrm{~N} 15,65 \mathrm{Y} 05,97 \mathrm{P} 40$

DOI. $10.1137 / 141000671$

\section{Contents}

I Scientific Computing Languages: The Julia Innovation 66

1.1 Julia Architecture and Language Design Philosophy . . . . . . . . . 67

${ }^{*}$ Received by the editors December 18, 2014; accepted for publication (in revised form) December 16, 2015; published electronically February 7, 2017.

http://www.siam.org/journals/sirev/59-1/100067.html

Funding: This work received financial support from the MIT Deshpande Center for Technological Innovation, the Intel Science and Technology Center for Big Data, the DARPA XDATA program, the Singapore MIT Alliance, an Amazon Web Services grant for JuliaBox, NSF awards CCF-0832997, DMS-1016125, and DMS-1312831, VMware Research, a DOE grant with Dr. Andrew Gelman of Columbia University for petascale hierarchical modeling, grants from Saudi Aramco thanks to Ali Dogru and Shell Oil thanks to Alon Arad, and a Citibank grant for High Performance Banking Data Analysis, Chris Mentzel, and the Gordon and Betty Moore Foundation.

${ }^{\dagger}$ Julia Computing, Inc. (jeff@juliacomputing.com, viral@juliacomputing.com).

$\ddagger$ CSAIL and Department of Mathematics, Massachusetts Institute of Technology, Cambridge, MA 02139 (edelman@math.mit.edu).

$\S$ New York University, New York, NY 10012, and Julia Computing, Inc. (stefan@juliacomputing. com). 
2 A Taste of Julia $\quad 68$

2.1 A Brief Tour . . . . . . . . . . . . . . . . . . . 68

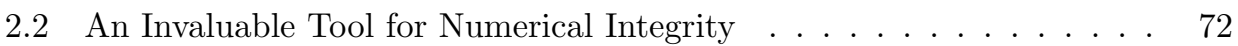

2.3 The Julia Community . . . . . . . . . . . . . . . . . . . . . . . . 74

3 Writing Programs With and Without Types 74

3.1 The Balance between Human and the Computer . . . . . . . . . . . . 74

3.2 Julia's Recognizable Types . . . . . . . . . . . . . . . . . . . . . 74

3.3 User's Own Types Are First Class Too . . . . . . . . . . . . . . . . . 75

3.4 Vectorization: Key Strengths and Serious Weaknesses . . . . . . . 76

3.5 Type Inference Rescues "For Loops" and So Much More . . . . . . . . 78

4 Code Selection: Run the Right Code at the Right Time 78

4.1 Multiple Dispatch . . . . . . . . . . . . . . . . . . 79

4.2 Code Selection from Bits to Matrices . . . . . . . . . . . . . . . . 81

4.2.1 Summing Numbers: Floats and Ints . . . . . . . . . . . . . 81

4.2.2 Summing Matrices: Dense and Sparse . . . . . . . . . . . . . 82

4.3 The Many Levels of Code Selection . . . . . . . . . . . . . . . . . . . 83

4.4 Is "Code Selection" Traditional Object Oriented Programming? . . . . 85

4.5 Quantifying the Use of Multiple Dispatch . . . . . . . . . . . . 86

4.6 Case Study for Numerical Computing . . . . . . . . . . . . . . 87

4.6.1 Determinant: Simple Single Dispatch . . . . . . . . . . . 88

4.6.2 A Symmetric Arrow Matrix Type . . . . . . . . . . . . . . 89

5 Leveraging Design for High Performance Libraries 9

5.1 Integer Arithmetic . . . . . . . . . . . . . . . . . . . . . . 90

5.2 A Powerful Approach to Linear Algebra . . . . . . . . . . . . . . . . . 91

5.2 .1 Matrix Factorizations . . . . . . . . . . . . . . . 91

5.2.2 User-Extensible Wrappers for BLAS and LAPACK . . . . . . . 92

5.3 High Performance Polynomials and Special Functions with Macros . . 93

5.4 Easy and Flexible Parallelism . . . . . . . . . . . . . . . . . . . . 94

5.5 Performance Recap . . . . . . . . . . . . . . . . . . . . . . . . . . 96

$\begin{array}{llr}6 \text { Conclusion } & 97\end{array}$

$\begin{array}{lr}\text { References } & 97\end{array}$

I. Scientific Computing Languages: The Julia Innovation. The original numerical computing language was Fortran, short for "Formula Translating System," released in 1957. Since those early days, scientists have dreamed of writing high-level, generic formulas and having them translated automatically into low-level, efficient code, tailored to the particular data types they need to apply the formulas to. Fortran made historic strides toward the realization of this dream, and its dominance in so many areas is a testament to its success.

The landscape of computing has changed dramatically over the years. Modern scientific computing environments such as Python [34], R [12], Mathematica [21], Octave [25], MATLAB [22], and Scilab [10], to name a few, have grown in popularity and fall under the general category known as dynamic languages or dynamically typed languages. In these programming languages, programmers write simple, high-level code without any mention of types like int, float, or double that pervade statically typed languages such as $\mathrm{C}$ and Fortran. 
Many researchers today work in dynamic languages. Still, C and Fortran remain the gold standard for performance for computationally intensive problems. As much as the dynamic language programmer misses out on performance, though, the $\mathrm{C}$ and Fortran programmer misses out on productivity. An unfortunate outcome is that the most challenging areas of numerical computing have benefited the least from the increased abstraction and productivity offered by higher-level languages. The consequences have been more serious than many realize.

Julia's innovation lies in its combination of productivity and performance. New users want a quick explanation as to why Julia is fast and want to know whether somehow the same "magic dust" could also be sprinkled on their favorite traditional scientific computing language. Julia is fast because of careful language design and the right combination of carefully chosen technologies that work very well with each other. This article demonstrates some of these technologies using a number of examples. Celeste serves as an example for readers interested in a large-scale application that leverages 8,192 cores on the Cori Supercomputer at Lawrence Berkeley National Laboratory [28].

Users interact with Julia through a standard REPL (real-eval-print loop environment such as Python, R, or MATLAB), by collecting commands in a .jl file, or by typing directly in a Jupyter (JUlia, PYThon, R) notebook $[15,30]$. We invite the reader to follow along at http://juliabox.com using Jupyter notebooks or by downloading Julia from http://julialang.org/downloads.

I.I. Julia Architecture and Language Design Philosophy. Many popular dynamic languages were not designed with the goal of high performance in mind. After all, if you wanted really good performance you would use a static language, or so said the popular wisdom. Only with the increasing need in the day-to-day life of scientific programmers for simultaneous productivity and performance has the need for high performance dynamic languages become pressing. Unfortunately, retrofitting an existing slow dynamic language for high performance is almost impossible, specifically in numerical computing ecosystems. This is because numerical computing requires performance-critical numerical libraries, which invariably depend on the details of the internal implementation of the high-level language, thereby locking in those internal implementation details. For example, you can run Python code much faster than the standard CPython implementation using the PyPy just-in-time (JIT) compiler, but PyPy is currently incompatible with NumPy and the rest of SciPy.

Another important point is that just because a program is available in $\mathrm{C}$ or Fortran, it may not run efficiently from the high-level language.

The best path to a fast, high-level system for scientific and numerical computing is to make the system fast enough that all of its libraries can be written in the high-level language in the first place. The JuMP.jl [20] package for mathematical programming and the Convex.jl [33] package for convex optimization are great examples of the success of this approach - in each case the entire library is written in Julia and uses many Julia language features described in this article.

The Two Language Problem. As long as the developers' language is harder to grasp than the users' language, numerical computing will always be hindered. This is an essential part of the design philosophy of Julia: all basic functionality must be possible to implement in Julia - never force the programmer to resort to using $\mathrm{C}$ or Fortran. Julia solves the two language problem. Basic functionality must be fast: integer arithmetic, for loops, recursion, floating-point operations, calling $\mathrm{C}$ functions, and manipulating C-like structs. While these features are not only important for 
numerical programs, without them you certainly cannot write fast numerical code. "Vectorization languages" like Python+NumPy, R, and MATLAB hide their for loops and integer operations, but they are still there, inside the $\mathrm{C}$ and Fortran, lurking beneath the thin veneer. Julia removes this separation entirely, allowing the high-level code to "just write a for loop" if that happens to be the best way to solve a problem.

We believe that the Julia programming language fulfills much of the Fortran dream: automatic translation of formulas into efficient executable code. It allows programmers to write clear, high-level, generic and abstract code that closely resembles mathematical formulas, yet produces fast, low-level machine code that has traditionally only been generated by static languages.

Julia's ability to combine these levels of performance and productivity in a single language stems from the choice of a number of features that work well with each other:

1. An expressive type system, allowing optional type annotations (section 3).

2. Multiple dispatch using these types to select implementations (section 4).

3. Metaprogramming for code generation (section 5.3).

4. A dataflow type inference algorithm allowing types of most expressions to be inferred $[2,4]$.

5. Aggressive code specialization against run-time types $[2,4]$.

6. JIT compilation $[2,4]$ using the LLVM compiler framework [18], which is also used by a number of other compilers such as Clang [6] and Apple's Swift [32].

7. Julia's carefully written libraries that leverage the language design, i.e., points 1 through 6 above (section 5).

Points 1, 2, and 3 above are features especially for the human user, and they are the focus of this paper. For details about the features related to language implementation and internals such as those in points 4,5 , and 6 , we direct the reader to our earlier work $[2,4]$. The feature in point 7 brings everything together to enable the building of high performance computational libraries in Julia.

Although a sophisticated type system is made available to the programmer, it remains unobtrusive in the sense that one is never required to specify types, and neither are type annotations necessary for performance. Type information flows naturally through the program due to dataflow type inference.

In what follows, we describe the benefits of Julia's language design for numerical computing, allowing programmers to more readily express themselves while also obtaining performance.

\section{A Taste of Julia.}

\section{I. A Brief Tour.}

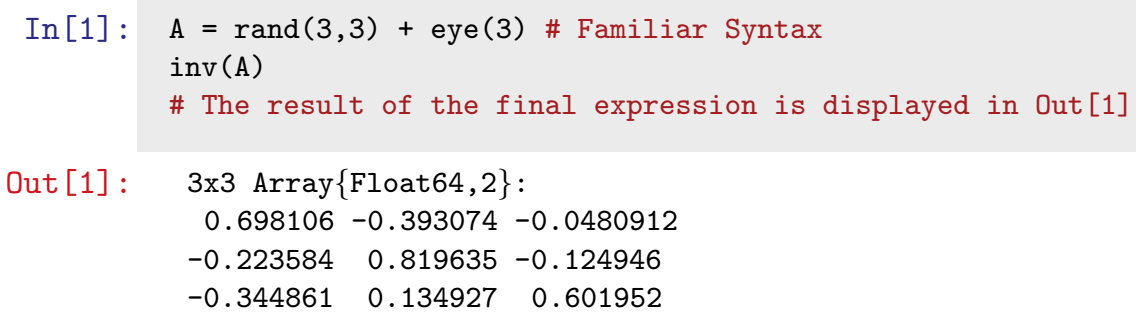

The output from the Julia prompt says that $A^{-1}$ is a two-dimensional matrix of size $3 \times 3$ and contains double precision floating-point numbers.

Indexing of arrays is performed with brackets with index origin 1. It is also possible to compute an entire array expression and then index into it, without assigning 
the expression to a variable:

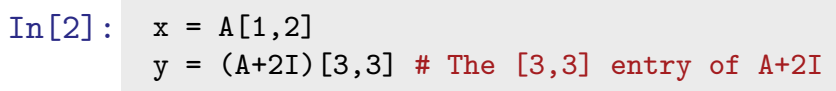

\section{Out [2]: $\quad 2.601952$}

In Julia, I is a built-in representation of the identity matrix, without any explicit forming of the identity matrix as is commonly done using commands such as "eye." ("eye," a homonym of "I," is used in such languages as MATLAB, Octave, Go's matrix library, Python's NumPy, and Scilab.)

Julia has symmetric tridiagonal matrices as a special type. For example, we may define Gil Strang's favorite matrix (the second-order difference matrix; see Figure 1) in a way that uses only $O(n)$ memory.

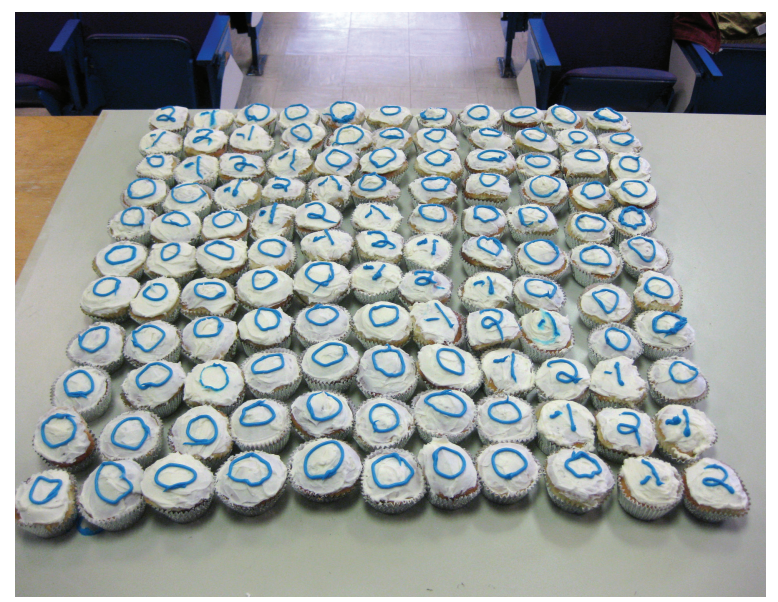

Fig. I Gil Strang's favorite matrix is $\operatorname{strang}(\mathrm{n})=\operatorname{SymTridiagonal}(2 *$ ones $(\mathrm{n})$, -ones(n-1)). Julia only stores the diagonal and off-diagonal. (Picture taken in Gil Strang's classroom.)

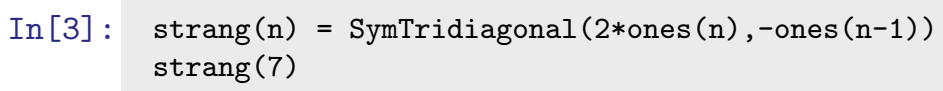

$\begin{array}{rrrrrrr}2.0 & -1.0 & 0.0 & 0.0 & 0.0 & 0.0 & 0.0 \\ -1.0 & 2.0 & -1.0 & 0.0 & 0.0 & 0.0 & 0.0 \\ 0.0 & -1.0 & 2.0 & -1.0 & 0.0 & 0.0 & 0.0 \\ 0.0 & 0.0 & -1.0 & 2.0 & -1.0 & 0.0 & 0.0 \\ 0.0 & 0.0 & 0.0 & -1.0 & 2.0 & -1.0 & 0.0 \\ 0.0 & 0.0 & 0.0 & 0.0 & -1.0 & 2.0 & -1.0 \\ 0.0 & 0.0 & 0.0 & 0.0 & 0.0 & -1.0 & 2.0\end{array}$

A commonly used notation to express the solution $x$ to the equation $A x=b$ is $\mathrm{A} \backslash \mathrm{b}$. If Julia knows that $A$ is a tridiagonal matrix, it uses an efficient $O(n)$ algorithm:

In [4]: $\quad$ strang (8) \ones (8) 


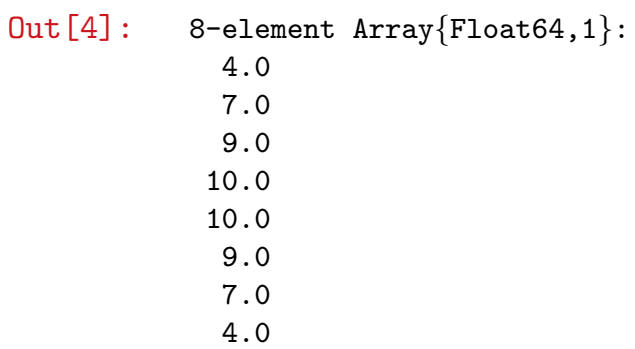

Note the Array\{ElementType, dims\} syntax. In the above example, the elements are 64-bit floats or Float64's. The 1 indicates it is a one-dimensional vector.

Consider the sorting of complex numbers. Sometimes it is handy to have a sort that generalizes the real sort. This can all be done by sorting first by the real part, and where there are ties, sort by the imaginary part. Other times it is handy to use the polar representation, which sorts by radius then by angle. By default, complex numbers are incomparable in Julia.

If a numerical computing language "hardwires" its sort to be one or the other, it misses an opportunity. A sorting algorithm need not depend on the details of what is being compared or how it is done so. One can abstract away these details, thereby enabling the reuse of a sorting algorithm for many different situations. One can specialize later. Thus, alphabetizing strings, sorting real numbers, and sorting complex numbers in two or more ways can all be done using the same code.

In Julia, one can turn a complex number w into an ordered pair of real numbers (a tuple of length 2) such as the Cartesian form (real (w), imag(w)) or the polar form (abs (w), angle $(\mathrm{w})$ ). Tuples are then compared lexicographically. The sort command takes an optional "less-than" operator, $1 t$, which is used to compare elements when sorting.

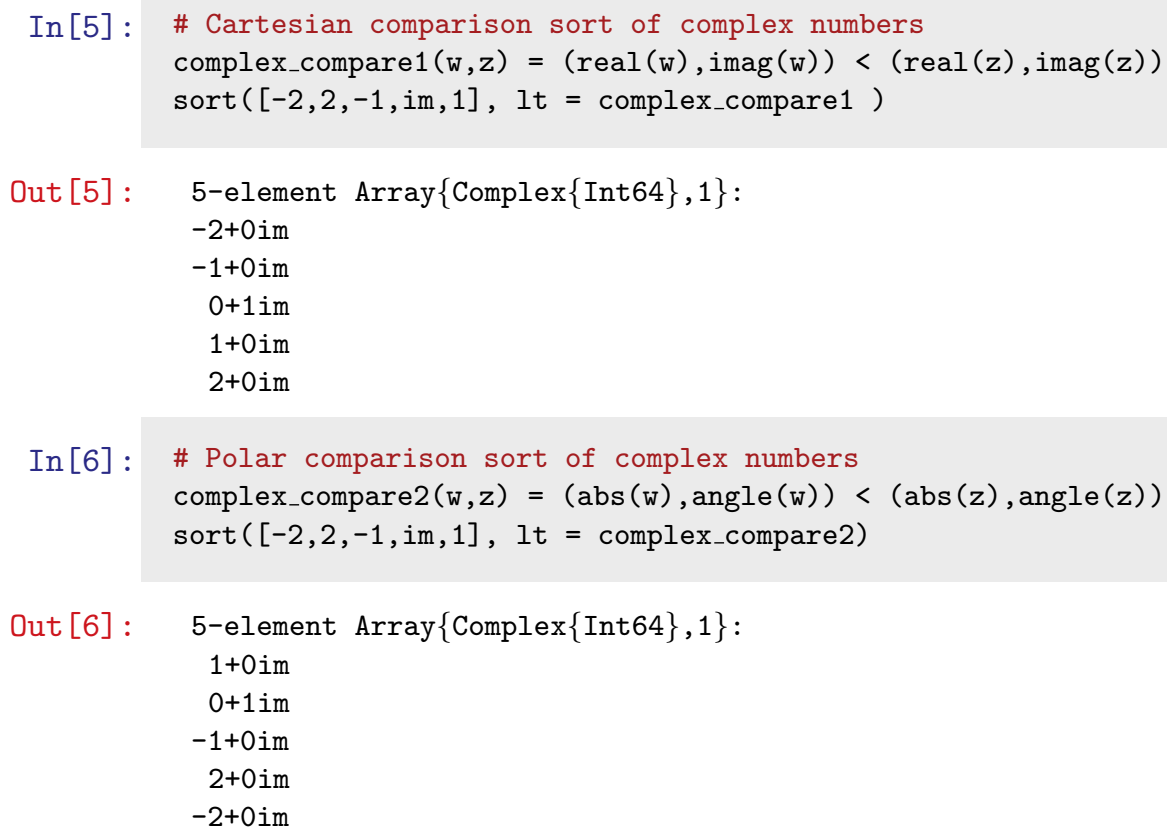

Copyright $@$ by SIAM. Unauthorized reproduction of this article is prohibited. 
To be sure, experienced computer scientists tend to suspect that there is nothing new under the sun. The $\mathrm{C}$ function qsort() takes a compar function, so there is nothing really new there. Python also has custom sorting with a key; MATLAB's sort is more basic. The real contribution of Julia, as will be fleshed out further in this article, is that its design allows custom sorting to be high performance, flexible, and comparable with implementations that are often written in C.

The next example that we have chosen for this introductory taste of Julia is a quick plot of Brownian motion, in two ways. The first uses the Python Matplotlib package for graphics, which is popular for users coming from Python or MATLAB. The second uses Gadfly.jl, another very popular package for plotting. Gadfly was built completely in Julia by Daniel Jones and was influenced by the much-admired "Grammar of Graphics" (see [36] and [35]). ${ }^{1}$ Many Julia users find Gadfly more flexible and prefer its aesthetics. Julia plots can also be manipulated interactively with sliders and buttons using its Interact.jl package. ${ }^{2}$ The Interact.jl package web page contains many examples of interactive visualizations. ${ }^{3}$
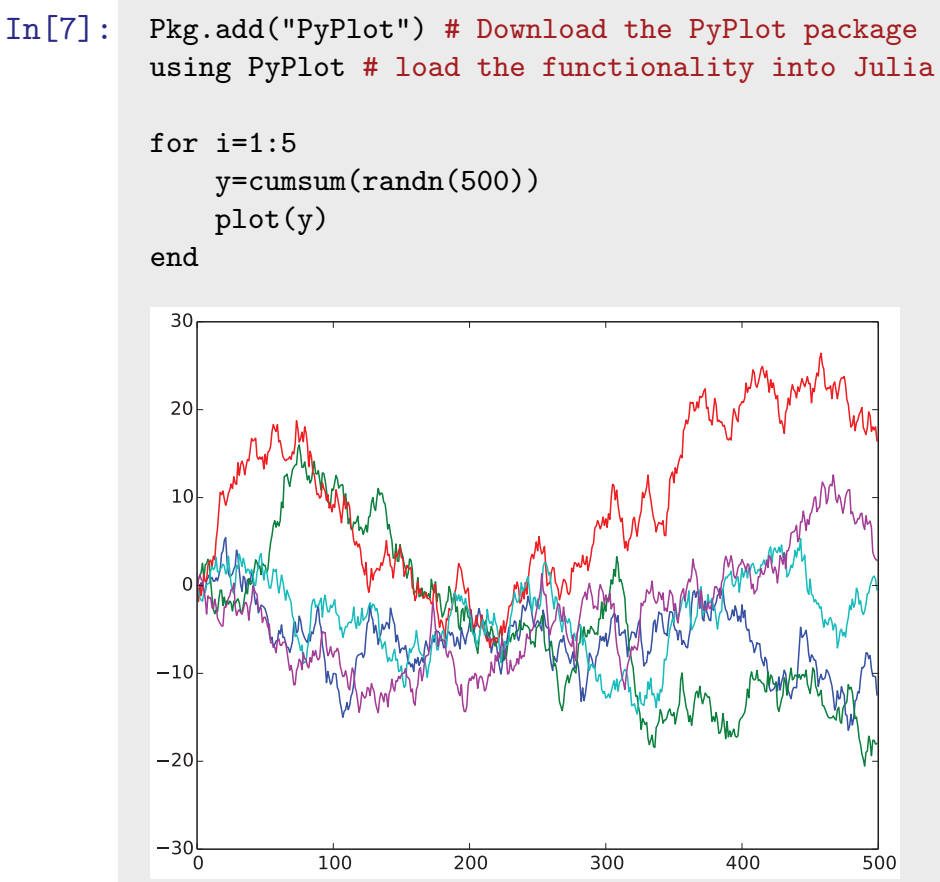

\footnotetext{
${ }^{1}$ See tutorial at http://gadflyjl.org

${ }^{2}$ https://github.com/JuliaLang/Interact.jl

${ }^{3}$ https://github.com/JuliaLang/Interact.jl/issues/36
} 
In [8]: Pkg.add("Gadfly") \# Download the Gadfly package

using Gadfly \# load the functionality into Julia

$\mathrm{n}=500$

$1=$ ["First", "Second", "Third"]

$c=$ [colorant"yellow", colorant"cyan", colorant"magenta"]

$\mathrm{p}=$ [layer $(\mathrm{x}=1: \mathrm{n}, \mathrm{y}=$ cumsum $(\operatorname{randn}(\mathrm{n}))$, Geom.line, Theme(default_color=i)) for $i$ in $c$ ]

labels=(Guide.xlabel ("Time"), Guide.ylabel ("Value"), Guide.title("Brownian Motion Trials"),

Guide.manual_color_key("Legend", 1, c))

Gadfly.plot (p..., labels...)

\section{Brownian Motion Trials}

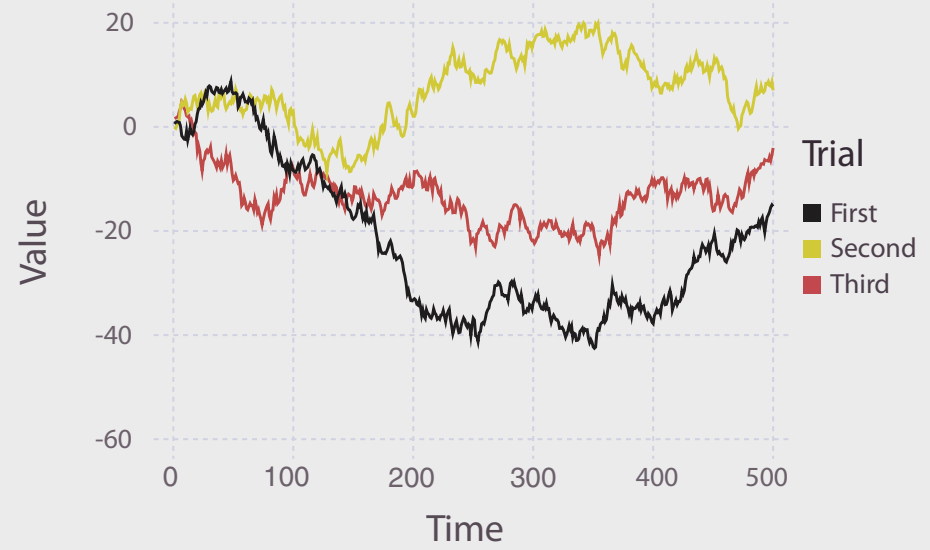

The ellipses on the last line of text above are known as a splat operator. The elements of the vector $\mathrm{p}$ and the tuple labels are inserted individually as arguments to the plot function.

2.2. An Invaluable Tool for Numerical Integrity. One popular feature of Julia is that it gives the user the ability to "kick the tires" of a numerical computation. We thank Velvel Kahan for the sage advice ${ }^{4}$ concerning the importance of this feature.

The idea is simple: a good engineer tests his or her code for numerical stability. In Julia this can be done by changing the IEEE rounding modes. There are five modes to choose from, yet most engineers silently choose only the RoundNearest mode default available in many numerical computing systems. If a difference is detected, one can also run the computation in higher precision. Kahan [16] writes:

Can the effects of roundoff upon a floating-point computation be assessed without submitting it to a mathematically rigorous and (if feasible at all) time-consuming error-analysis? In general, No....

\footnotetext{
${ }^{4}$ Personal communication, January 2013, in the Kahan home, Berkeley, California.
} 
Though far from foolproof, rounding every inexact arithmetic operation (but not constants) in the same direction for each of two or three directions besides the default To Nearest is very likely to confirm accidentally exposed hypersensitivity to roundoff. When feasible, this scheme offers the best Benefit/Cost ratio.

As an example, we round a $15 \times 15$ Hilbert-like matrix and take the [1,1] entry of the inverse computed in various roundoff modes. The radically different answers dramatically indicate the numerical sensitivity to roundoff. We have even noticed that slight changes to LAPACK lead to radically different answers. Most likely you will see different numbers when you run this code due to its very high sensitivity to roundoff errors.

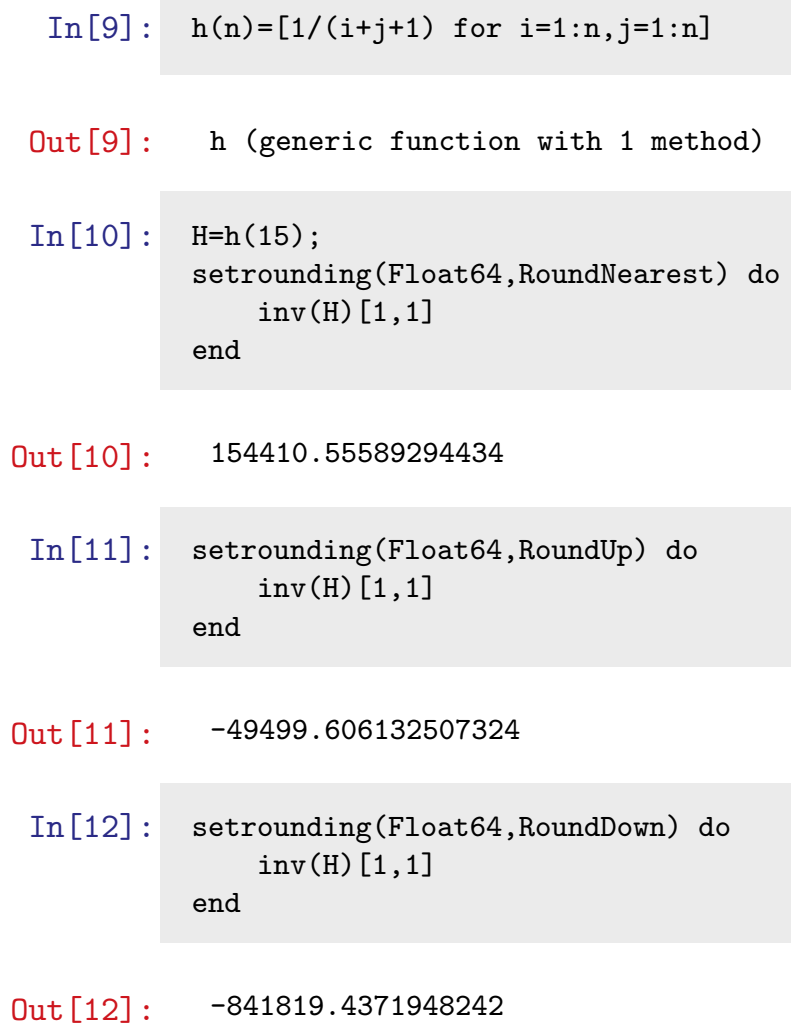

With 300 bits of precision, we obtain

In [13]: with_bigfloat_precision(300) do end

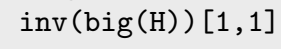

Out [13]: $\quad-2.09397179250746270128280174214489516162708857703714959763232689047153$ $50765882491054998376252 \mathrm{e}+03$

Note that this is the $[1,1]$ entry of the inverse of the rounded Hilbert-like matrix, not the inverse of the exact Hilbert-like matrix, whose entry would be exactly 1,387,200. Also, the Float64 results are sensitive to the BLAS [19] and LAPACK [1] 
libraries, and may differ on different machines with different versions of Julia. For extended precision, Julia uses the MPFR library [9].

2.3. The Julia Community. Julia has been under development since 2009, and a public release was announced in February of 2012. It is an active open source project with over 500 contributors and is available under the MIT License [23] for open source software. Over 2 million unique visitors have visited the Julia website since then, and Julia has now been adopted as a teaching tool in dozens of universities around the world. ${ }^{5}$ The community has contributed over 1200 Julia packages. While it was nurtured at the Massachusetts Institute of Technology, it is really the contributions from experts around the world that make it a joy to use for numerical computing. It is also recognized as a general purpose computing language, unlike traditional numerical computing systems, allowing it to be used not only to prototype numerical algorithms, but also to deploy those algorithms and even serve results to the rest of the world. A great example of this is Shashi Gowda's Escher.jl package, ${ }^{6}$ which makes it possible for Julia programmers to build beautiful interactive websites in Julia and serve up the results of a Julia computation from the web server, without any knowledge of HTML or JavaScript. Another such example is "Sudoku-as-a-Service,"7 by Iain Dunning, where a Sudoku puzzle is solved using the optimization capabilities of the JuMP.jl Julia package [20] and made available as a web service. This is exactly why Julia is being increasingly deployed in production environments in businesses, as is seen in various talks at JuliaCon. ${ }^{8}$ These use cases utilize Julia's capabilities not only for mathematical computation, but for building web APIs, database access, and much more.

\section{Writing Programs With and Without Types.}

3.I. The Balance between Human and the Computer. Graydon Hoare, author of the Rust programming language [29], defined programming languages succinctly in an essay on Interactive Scientific Computing [11]:

Programming languages are mediating devices, interfaces that try to strike a balance between human needs and computer needs. Implicit in that is the assumption that human and computer needs are equally important, or need mediating.

A program consists of data and operations on data. Data is not just the input file, but everything that is held - an array, a list, a graph, a constant - during the life of the program. The more the computer knows about this data, the better it is at executing operations on it. Types are exactly this metadata. Describing this metadata, the types, takes real effort for the human. Statically typed languages such as C and Fortran are at one extreme, where all types must be defined and are statically checked during the compilation phase. The result is excellent performance. Dynamically typed languages dispense with type definitions, which leads to greater productivity but lower performance as the compiler and the runtime cannot benefit from the type information that is essential to producing fast code. Can we strike a balance between the human's preference to avoid types and the computer's need to know?

3.2. Julia's Recognizable Types. Many users of Julia may never need to know about types for performance. Julia's type inference system often does all the work, giving performance without type declarations.

\footnotetext{
${ }^{5}$ http://julialang.org/community

${ }^{6}$ https://github.com/shashi/Escher.jl

${ }^{7}$ http://iaindunning.com/2013/sudoku-as-a-service.html

${ }^{8} \mathrm{http}: / /$ www.juliacon.org
} 
Julia's design allows for the gradual learning of concepts, where users start in a manner that is familiar to them and, over time, learn to structure programs in the "Julian way" - a term that implies well-structured readable high performance Julia code. Julia users coming from other numerical computing environments have a notion that data may be represented as matrices that may be dense, sparse, symmetric, triangular, or of some other kind. They may also, though not always, know that elements in these data structures may be single or double precision floating-point numbers, or integers of a specific width. In more general cases, the elements within data structures may be other data structures. We introduce Julia's type system using matrices and their number types:

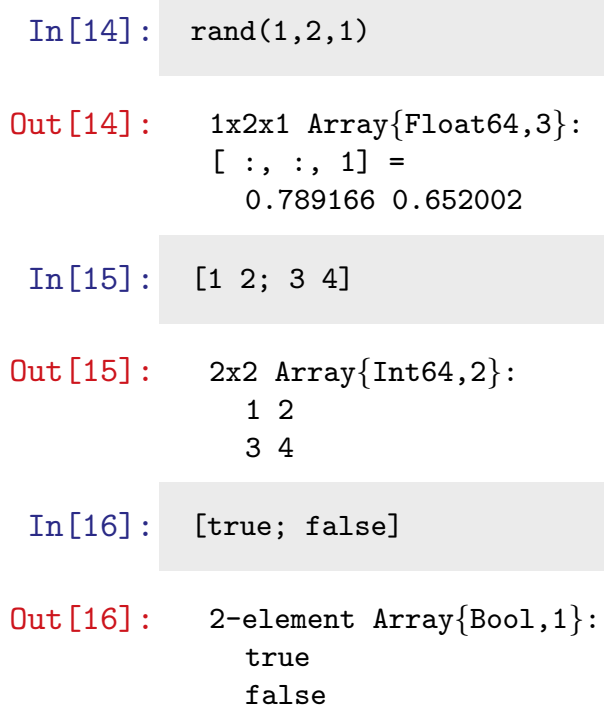

We see a pattern in the examples above. Array\{T,ndims\} is the general form of the type of a dense array with ndims dimensions whose elements themselves have a specific type $\mathrm{T}$, which is of type double precision floating point in the first example, a 64-bit signed integer in the second, and a boolean in the third example. Therefore, Array $\{\mathrm{T}, 1\}$ is a one-dimensional vector (first class objects in Julia) with element type $\mathrm{T}$ and Array $\{\mathrm{T}, 2\}$ is the type for two-dimensional matrices.

It is useful to think of an array as a generic $\mathrm{N}$-dimensional object that may contain elements of any type $\mathrm{T}$. Thus, $\mathrm{T}$ is a type parameter for an array that can take on many different values. Similarly, the dimensionality of the array ndims is also a parameter for the array type. This generality makes it possible to create arrays of arrays. For example, using Julia's array comprehension syntax, we create a two-element vector containing $2 \times 2$ identity matrices:

$\operatorname{In}[17]: \quad a=[$ eye(2) for $i=1: 2]$

Out [17]: 2-element Array $\{$ Array $\{$ Float64, 2\}, 1\}:

3.3. User's Own Types Are First Class Too. Many dynamic languages for numerical computing have traditionally contained an asymmetry, with built-in types having much higher performance than any user-defined types. This is not the case 
with Julia, where there is no meaningful distinction between user-defined and "builtin" types.

We have mentioned so far a few number types and two matrix types: Array\{T , 2\}, the dense array with element type $\mathrm{T}$, and SymTridiagonal $\{\mathrm{T}\}$, the symmetric tridiagonal with element type T. There are also other matrix types for other structures including SparseMatrixCSC (compressed sparse columns), Hermitian, Triangular, Bidiagonal, and Diagonal. Julia's sparse matrix type has an added flexibility, that it can go beyond storing just numbers as nonzeros, and can instead store any other Julia type as well. The indices in SparseMatrixCSC can also be represented as integers of any width (16-bit, 32-bit, or 64-bit). All these different matrix types, available as built-in types to a user downloading Julia, are implemented completely in Julia and are in no way any more or less special than any other types a user may define in their own program.

For demonstration purposes, we now create a symmetric arrow matrix type that contains a diagonal and the first row A $[1,2: \mathrm{n}]$. Oce could also throw an ArgumentError if the ev vector was not one shorter in length than the $\mathrm{dv}$ vector.

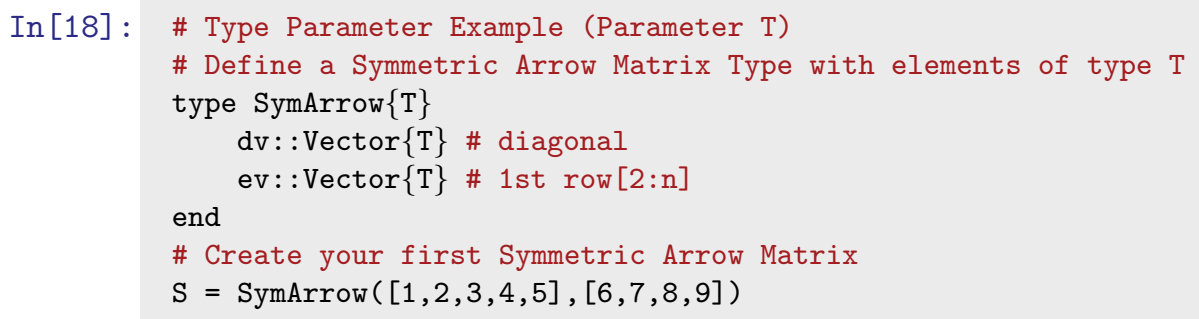

Out [18]: $\quad$ SymArrow $\{\operatorname{Int} 64\}([1,2,3,4,5],[6,7,8,9])$

The parameter in the array refers to the type of each element of the array. Code can and should be written independently of the type of each element.

In section 4.6.2, we develop the symmetric arrow example much further. The SymArrow matrix type contains two vectors, one each for the diagonal and the first row, and these vectors contain elements of type T. In the type definition, the type SymArrow is parametrized by the type of the storage element T. By doing so, we have created a generic type, which refers to a universe of all arrow matrices containing elements of all types. The matrix $\mathrm{S}$ is an example where $\mathrm{T}$ is Int64. When we write functions in section 4.6.2 that operate on arrow matrices, those functions themselves will be generic and applicable to the entire universe of arrow matrices we have defined here.

Julia's type system allows for abstract types, concrete "bits" types, composite types, and immutable composite types. All of these types can have parameters and users may even write programs using unions of them. We refer the reader to full details about Julia's type system in the types chapter in the Julia manual. ${ }^{9}$

3.4. Vectorization: Key Strengths and Serious Weaknesses. Users of traditional high-level computing languages know that vectorization improves performance. Do most users know exactly why vectorization is so useful? It is precisely because,

${ }^{9}$ See http://docs.julialang.org/en/latest/manual/types/ 
by vectorizing, the user has promised the computer that the type of an entire vector of data matches the very first element. This is an example where users are willing to provide type information to the computer without even knowing that is what they are doing. Hence, it is an example of a strategy that balances the computer's needs with the human's.

From the computer's viewpoint, vectorization means that operations on data happen largely in sections of the code where types are known to the runtime system. The runtime has no idea about the data contained in an array until it encounters the array. Once encountered, the type of the data within the array is known, and this knowledge is used to execute an appropriate high performance kernel. Of course, what really occurs at runtime is that the system figures out the type and then reuses that information through the length of the array. As long as the array is not too small, all the extra work incurred in gathering type information and acting upon it at run time is amortized over the entire operation.

The downside of this approach is that the user can achieve high performance only with built-in types. User-defined types end up being dramatically slower. The restructuring for vectorization is often unnatural, and at times not possible. We illustrate this with an example of a cumulative sum computation. Note that due to the size of the problem, the computation is memory bound, and one does not observe the case with complex arithmetic to be twice as slower than the real case, even though it is performing twice as many floating point operations.

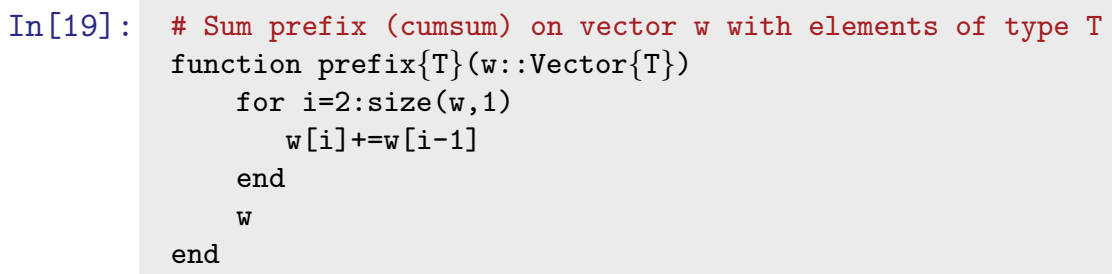

We execute this code on a vector of double precision real numbers and double precision complex numbers and observe something that may seem remarkable: similar run times in each case.

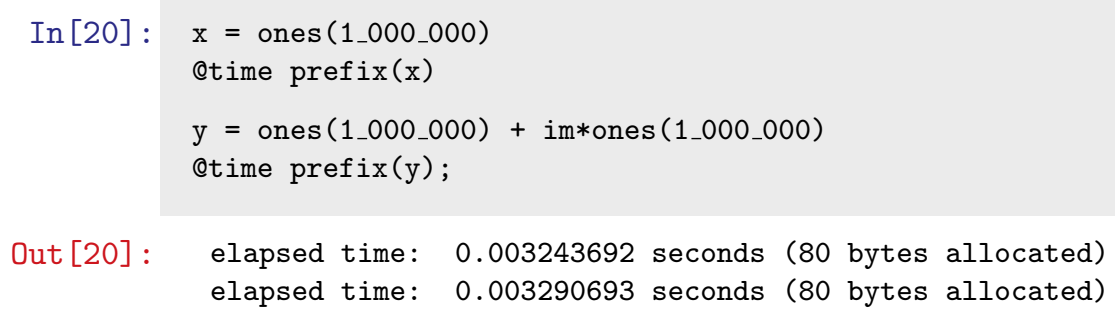

This simple example is difficult to vectorize, and hence is often provided as a builtin function in many numerical computing systems. In Julia, the implementation is very similar to the snippet of code above and runs at speeds similar to C. While Julia users can write vectorized programs as in any other dynamic language, vectorization is not a prerequisite for performance. This is because Julia strikes a different balance 
between the human and the computer when it comes to specifying types. Julia allows optional type annotations, which are essential when writing libraries but not for enduser programs that are exploring algorithms or a dataset.

Generally, in Julia, type annotations are not used for performance, but purely for code selection (see section 4). If the programmer annotates their program with types, the Julia compiler will use that information. However, in general, user code often includes minimal or no type annotations, and the Julia compiler automatically infers the types.

3.5. Type Inference Rescues "For Loops" and So Much More. A key component of Julia's ability to combine performance with productivity in a single language is its implementation of dataflow type inference $[24,17,4]$. Unlike type inference algorithms for static languages, this algorithm is tailored to the way dynamic languages work: the typing of code is determined by the flow of data through it. The algorithm works by walking through a program, starting with the types of its input values, and "abstractly interpreting" it: instead of applying the code to values, it applies the code to types, following all branches concurrently and tracking all possible states the program could be in, including all the types each expression could assume.

The dataflow type inference algorithm allows programs to be automatically annotated with type bounds without forcing the programmer to explicitly specify types. Yet, in dynamic languages it is possible to write programs which inherently cannot be concretely typed. In such cases, dataflow type inference provides what bounds it can, but these may be trivial and useless - i.e., they may not narrow down the set of possible types for an expression at all. However, the design of Julia's programming model and standard library are such that a majority of expressions in typical programs can be concretely typed.

A lesson of the numerical computing languages is that one must learn to vectorize to get performance. The mantra is "for loops" are bad, vectorization is good. Indeed one can find the following mantra on p.72 of the 1998 Getting Started with MATLAB manual (and other editions):

\section{Experienced MATLAB users like to say "Life is too short to spend writing for loops."}

It is not that "for loops" are inherently slow in themselves. The slowness comes from the fact that in the case of most dynamic languages, the system does not have access to the types of the variables within a loop. Since programs often spend much of their time doing repeated computations, the slowness of a particular operation due to lack of type information is magnified inside a loop. This leads to users often talking about "slow for loops" or "loop overhead."

4. Code Selection: Run the Right Code at the Right Time. Code selection or code specialization from one point of view is the opposite of the code reuse enabled by abstraction. Ironically, viewed another way, it enables abstraction. Julia allows users to overload function names and select code based on argument types. This can happen at the highest and lowest levels of the software stack. Code specialization lets us optimize for the details of the case at hand. Code abstraction lets calling codes, even those not yet written or perhaps not even imagined, work on structures that may not have been envisioned by the original programmer. 
We see this as the ultimate realization of the famous 1908 quip that

Mathematics is the art of giving the same name to different things. ${ }^{10}$

by noted mathematician Henri Poincaré.

In the next section we provide examples of how "plus" can apply to so many objects, such as floating-point numbers or integers. It can also apply to sparse and dense matrices. Another example is the use of the same name, "det," for determinant, for the very different algorithms that apply to very different matrix structures. The use of overloading not only for single argument functions, but also for multiple argument functions, is already a powerful abstraction.

4.I. Multiple Dispatch. Multiple dispatch is the selection of a function implementation based on the types of each argument of the function. It is not only a nice notation to remove a long list of "case" statements, but is also part of the reason for Julia's speed. It is expressed in Julia by annotating the type of a function argument in a function definition with the following syntax: argument: :Type.

Mathematical notations that are often used in print can be difficult to employ in programs. For example, we can teach the computer some natural ways to multiply numbers and functions. Suppose that $a$ and $t$ are scalars, and $f$ and $g$ are functions, and we wish to define the following operations:

1. Number $\mathbf{x}$ Function = scale output: $a * g$ is the function that takes $x$ to $a * g(x)$;

2. Function $\mathrm{x}$ Number $=$ scale argument $: f * t$ is the function that takes $x$ to $f(t x)$; and

3. Function $\mathrm{x}$ Function $=$ composition of functions: $f * g$ is the function that takes $x$ to $f(g(x))$.

If you were a mathematician who does not program, you would not see the fuss. If, however, you wanted to implement this in your favorite computer language, you might immediately see the benefit. In Julia, multiple dispatch makes all three uses of

\footnotetext{
${ }^{10} \mathrm{~A}$ few versions of Poincaré's quote are relevant to Julia's power of abstraction and numerical computing. They are worth pondering:

It is the harmony of the different parts, their symmetry, and their happy adjustment; it is, in a word, all that introduces order, all that gives them unity, that enables us to obtain a clear comprehension of the whole as well as of the parts. Elegance may result from the feeling of surprise caused by the unlooked-for occurrence of objects not habitually associated. In this, again, it is fruitful, since it discloses thus relations that were until then unrecognized. Mathematics is the art of giving the same names to different things.

(http://www.nieuwarchief.nl/serie5/pdf/naw5-2012-13-3-154.pdf)

One example has just shown us the importance of terms in mathematics; but I could quote many others. It is hardly possible to believe what economy of thought, as Mach used to say, can be effected by a well-chosen term. I think I have already said somewhere that mathematics is the art of giving the same name to different things. It is enough that these things, though differing in matter, should be similar in form, to permit of their being, so to speak, run in the same mould. When language has been well chosen, one is astonished to find that all demonstrations made for a known object apply immediately to many new objects: nothing requires to be changed, not even the terms, since the names have become the same.
}

(http://www-history.mcs.st-andrews.ac.uk/Extras/Poincare_Future.html) 


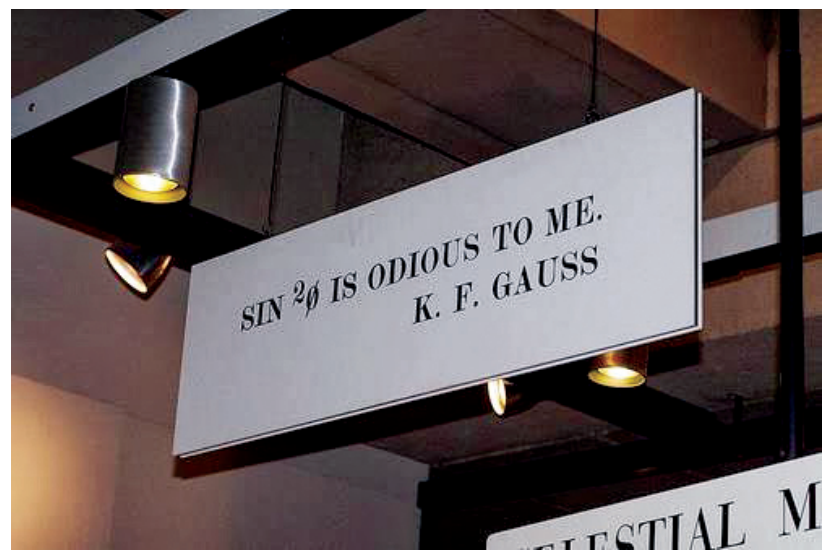

Fig. 2 Gauss quote hanging from the ceiling of the longstanding Boston Museum of Science Mathematica Exhibit.

"*" easy to express:

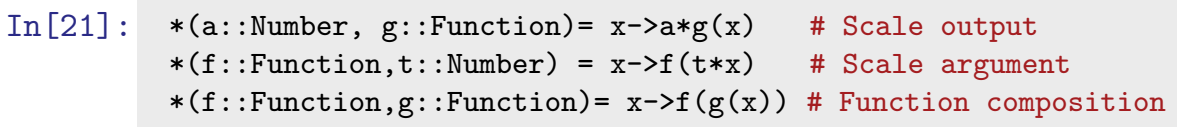

Here, multiplication is dispatched by the type of its first and second arguments. It is implemented in the usual way if both are numbers, but there are three new ways if one, the other, or both are functions.

These definitions exist as part of a larger system of generic definitions, which can be reused by later definitions. Consider the case of the mathematician Gauss's preference for $\sin ^{2} \phi$ to refer to $\sin (\sin (\phi))$ and not $\sin (\phi)^{2}$ (writing " $\sin ^{2}(\phi)$ is odious to me, even though Laplace made use of it." (see Figure 2). By defining $*(f:$ :Function, $\mathrm{g}:$ :Function $)=\mathrm{x}>\mathrm{f}(\mathrm{g}(\mathrm{x})),\left(\mathrm{f}^{\wedge} 2\right)(\mathrm{x})$ automatically computes $f(f(x))$, as Gauss wanted. This is a consequence of a generic definition that evaluates $x^{\wedge} 2$ as $x * x$ no matter how $\mathrm{x} * \mathrm{x}$ is defined.

This paradigm is a natural fit for numerical computing, since so many important operations involve interactions among multiple values or entities. Binary arithmetic operators are obvious examples, but many other uses abound. The fact that the compiler can pick the sharpest matching definition of a function based on its input types helps achieve higher performance, by keeping the code execution paths tight and minimal.

We have not seen this elsewhere in the literature but it seems worthwhile to point out four dispatch possibilities:

1. Static single dispatch (not done).

2. Static multiple dispatch (frequent in static languages, e.g., $\mathrm{C}++$ overloading).

3. Dynamic single dispatch (MATLAB's object oriented system might fall into this category, though it has its own special characteristics).

4. Dynamic multiple dispatch (usually just called multiple dispatch).

In section 4.4 we discuss the comparison with traditional object oriented approaches. Class-based object oriented programming could reasonably be called dy- 
namic single dispatch, and overloading could reasonably be called static multiple dispatch. Julia's dynamic multiple dispatch approach is more flexible and adaptable while still retaining powerful performance capabilities. Julia programmers often find that dynamic multiple dispatch makes it easier to structure their programs in ways that are closer to the underlying science.

4.2. Code Selection from Bits to Matrices. Julia uses the same mechanism for code selection at all levels, from the top to the bottom.

\begin{tabular}{|c|c|c|}
\hline f & Function & Operand Types \\
\hline Low-Level "+" & Add Numbers & \{Float , Int \\
High-Level "+" & Add Matrices & \{Dense Matrix , Sparse Matrix $\}$ \\
" $*$ Scale or Compose & \{Function, Number $\}$ \\
\hline
\end{tabular}

4.2.I. Summing Numbers: Floats and Ints. We begin at the lowest level. Mathematically, integers are thought of as being special real numbers, but on a computer, an Int and a Float have two very different representations. Ignoring for a moment that there are even many choices of Int and Float representations, if we add two numbers, code selection based on numerical representation is taking place at a very low level. Most users are blissfully unaware of this code selection, because it is hidden somewhere that is usually off-limits. Nonetheless, one can follow the evolution of the high-level code all the way down to the assembler level, which will ultimately reveal an ADD instruction for integer addition and, for example, the $\mathrm{AVX}^{11}$ instruction VADDSD $^{12}$ for floating-point addition in the language of x 86 assembly level instructions. The point here is that ultimately two different algorithms are being called, one for a pair of Ints and one for a pair of Floats.

Figure 3 takes a close look at what a computer must do to perform $x+y$ depending on whether (x,y) is (Int,Int), (Float,Float), or (Int,Float), respectively. In the first case, an integer add is called, while in the second case a float add is called. In the last case, a promotion of the int to float is implemented with the $\mathrm{x} 86$ instruction VCVTSI2SD, ${ }^{13}$ and then the float add follows.

It is instructive to build a Julia simulator in Julia itself.

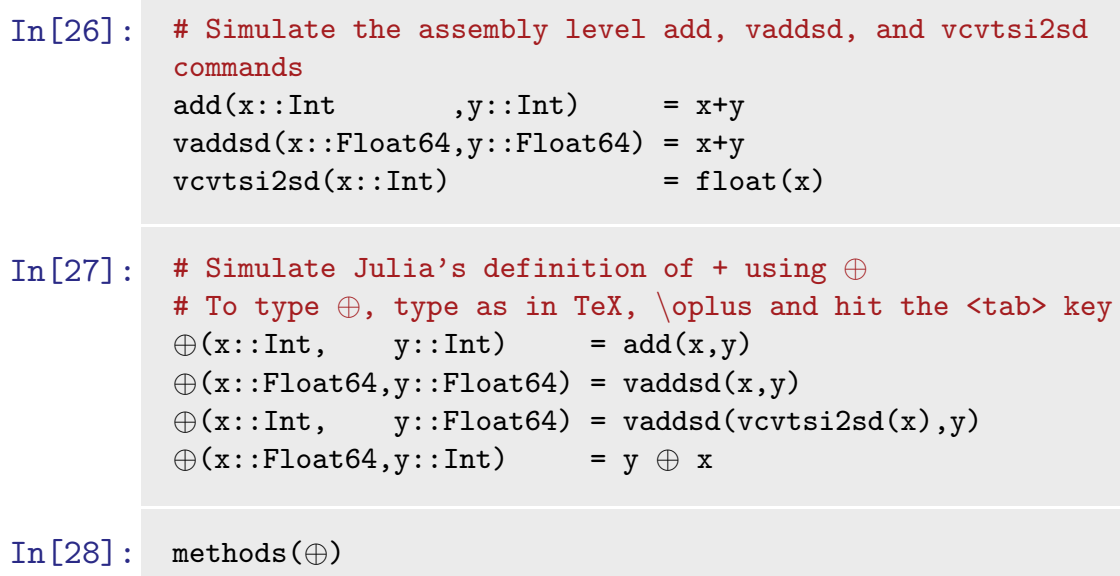

${ }^{11} \mathrm{AVX}$ : Advanced Vector eXtension to the $\mathrm{x} 86$ instruction set

${ }^{12}$ VADDSD: Vector ADD Scalar Double-precision

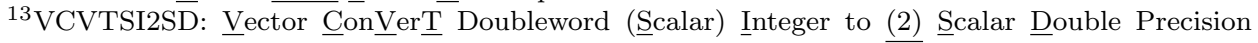
Floating-Point Value 


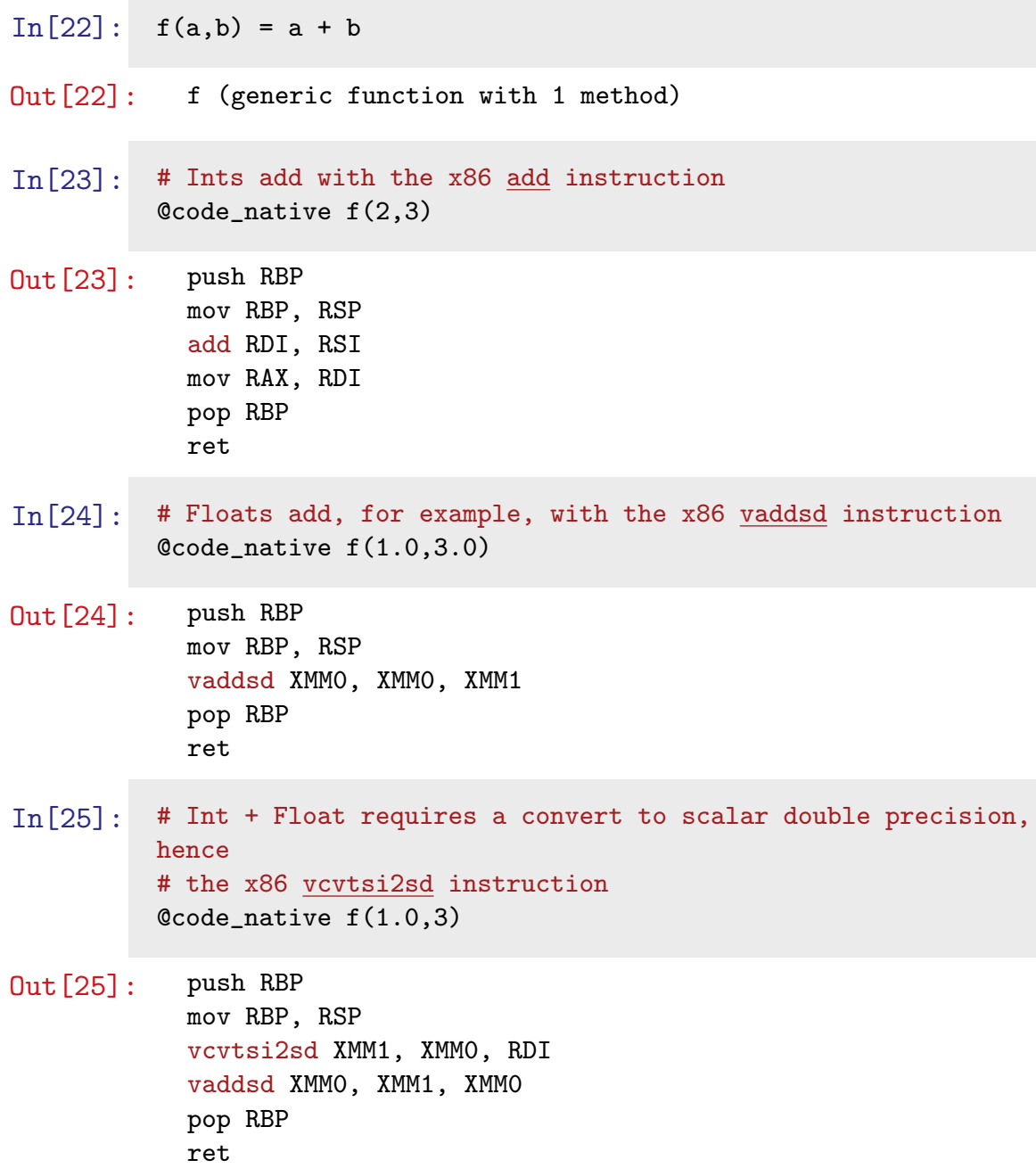

Fig. 3 While assembly code may seem intimidating, Julia disassembles readily. Armed with the code_native command in Julia and perhaps a good list of assembler commands such as may be found on http://docs.oracle.com/cd/E36784_\$01\$/pdf/E36859.pdf or http://en. wikipedia.org/wiki/X86_instruction_listings, one can really learn to see the details of code selection in action at the lowest levels. More importantly, one can begin to understand that Julia is fast because the assembly code produced is so tight.

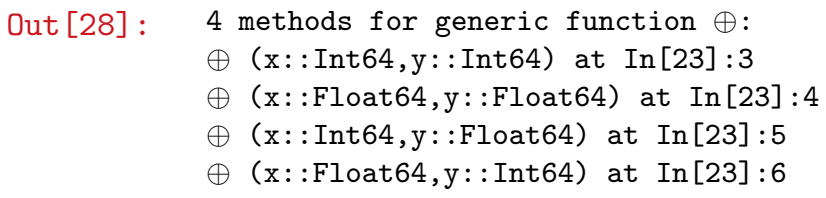

4.2.2. Summing Matrices: Dense and Sparse. We now move to a much higher level: matrix addition. The versatile "+" symbol lets us add matrices. On a computer, dense matrices are (usually) contiguous blocks of data with a few parameters 
attached, while sparse matrices (which may be stored in many ways) require storage of index information one way or another. If we add two matrices, code selection must take place depending on whether the summands are (dense,dense), (dense,sparse), (sparse,dense), or (sparse,sparse).

While this is at a much higher level, the basic pattern is unmistakably the same as that of section 4.2.1. We show how to use a dense algorithm in the implementation of $\oplus$ when either $A$ or $B$ (or both) are dense. A sparse algorithm is used when both $A$ and $B$ are sparse.

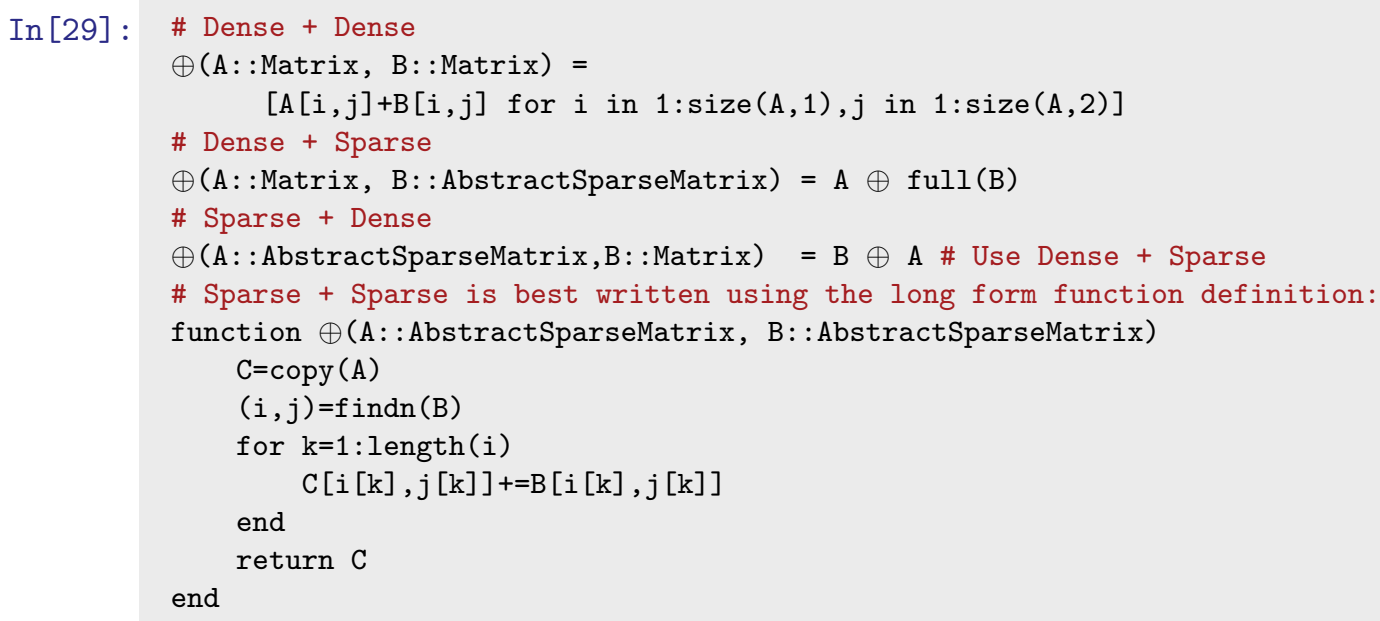

We have eight methods for the function $\oplus$, four for the low-level sum, and four more for the high level:

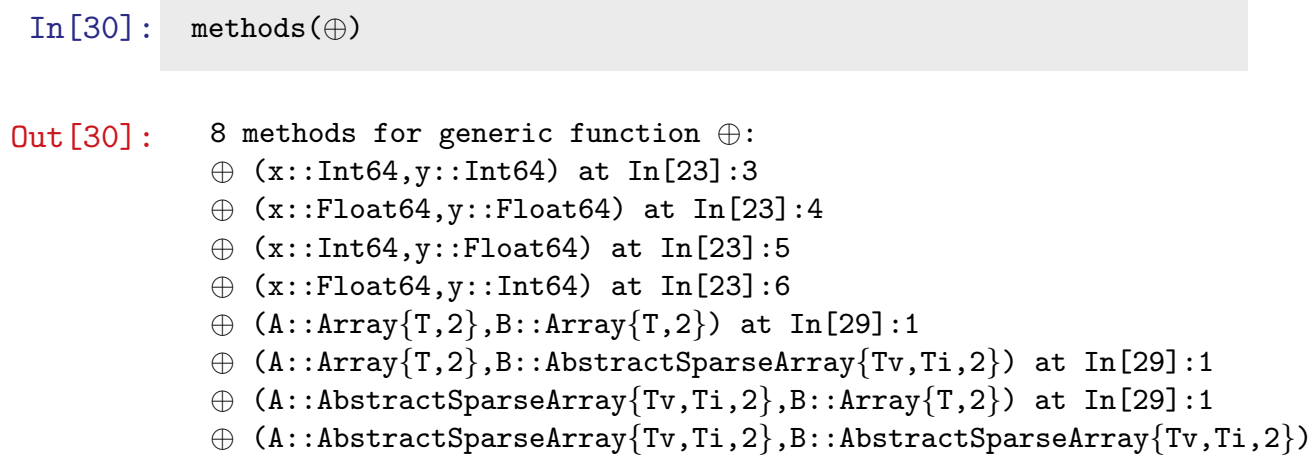

4.3. The Many Levels of Code Selection. In Julia, as in mathematics, functions are as important as the data they operate on, their arguments, and perhaps even more so. We can create a new function foo and gave it six definitions depending on the combination of types. In the following example we sensitize unfamiliar readers with terms from computer science language research. It is not critical that these terms be understood all at once. 


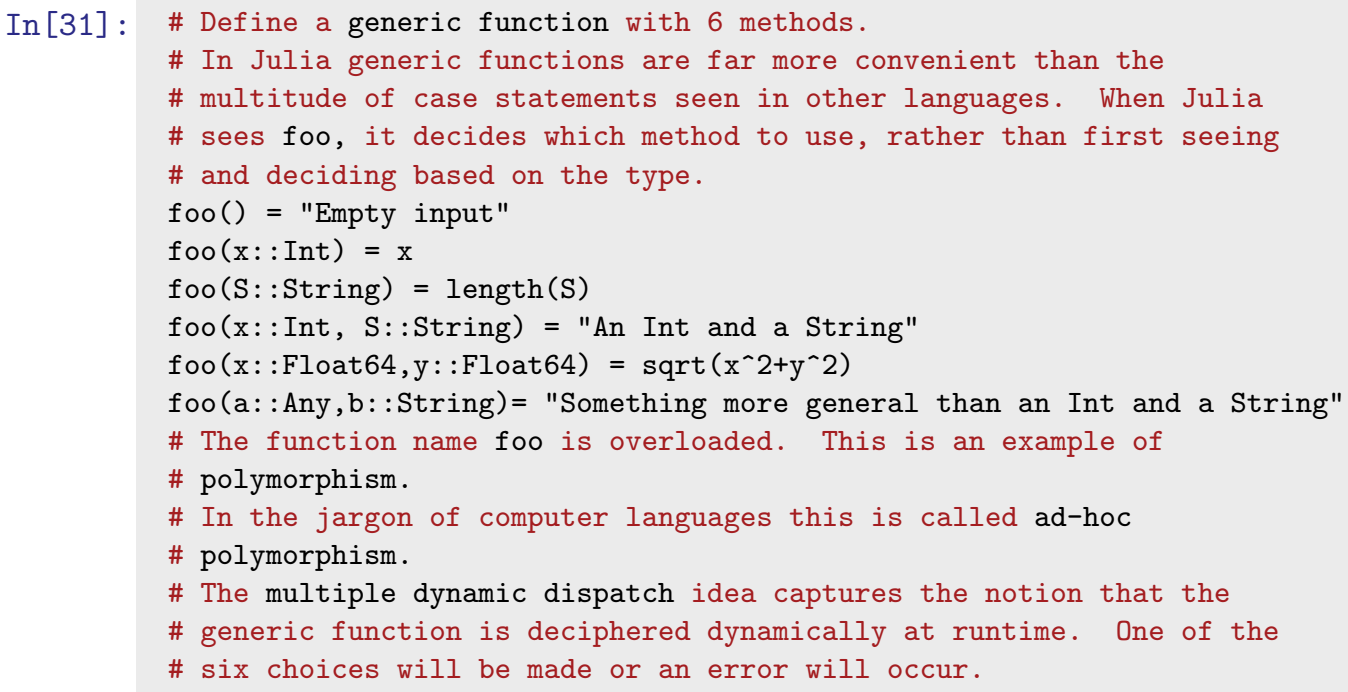

Out [31]: foo (generic function with 6 methods)

Any one instance of foo is a method. The collection of six methods is referred to as a generic function. The word "polymorphism" refers to the use of the same name (foo, in this example) for functions with different types. Contemplating the Poincaré quote in footnote 5 , it is handy to reason about everything to which you are giving the same name. In actual coding, one tends to use the same name when the abstraction makes a great deal of sense, so we use the same name "+" for ints, floats, dense, and sparse matrices. Methods are grouped into generic functions.

While mathematics is the art of giving the same name to seemingly different things, a computer eventually has to execute the right program in the right circumstance. Julia's code selection operates at multiple levels in order to translate a user's abstract ideas into efficient execution. A generic function can operate on several arguments, and the method with the most specific signature matching the arguments is invoked. It is worth crystallizing some key aspects of this process:

1. The same name can be used for different functions in different circumstances. For example, select may refer to the selection algorithm for finding the $k$ th smallest element in a list, or to select records in a database query, or simply to a user-defined function in a user's own program. Julia's namespaces allow the usage of the same vocabulary in different circumstances in a simple way that makes programs easy to read.

2. A collection of functions that represent the same idea but operate on different structures are naturally referred to by the same name. The particular method called is based entirely on the types of all the arguments - this is multiple dispatch. The function det may be defined for all matrices at an abstract level. However, for reasons of efficiency, Julia defines different methods for different types of matrices, depending on whether they are dense or sparse or have a special structure such as diagonal or tridiagonal.

3. Within functions that operate on the same structure, there may be further differences based on the different types of data contained within. For example, whether the input is a vector of Float64 values or Int32 values, the norm 


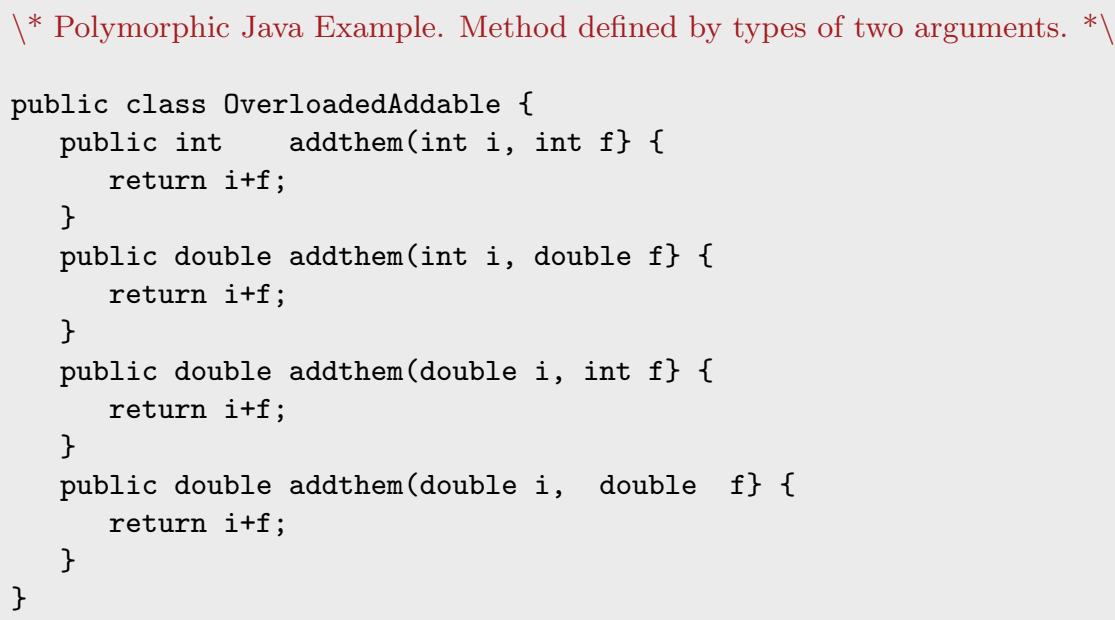

Fig. 4 Advantages of Julia: It is true that this Java code is polymorphic, based on the types of the two arguments. ("Polymorphism" means the use of the same name for a function that may have different type arguments.) However, in Java if the method addthem is called, the types of the arguments must be known at compile time. This is static dispatch. Java is also encumbered by encapsulation: in this case addthem is encapsulated inside the OverloadedAddable class. While this is considered a safety feature in the Java culture, it becomes a burden for numerical computing.

is computed in exactly the same way, with a common body of code, but the compiler is able to generate different executable code from the abstract specification.

4. Julia uses the same mechanism of code selection at the lowest and highest levels, whether it is performing operations on matrices or operations on bits. As a result, Julia is able to optimize the whole program, picking the right method at the right time, either at compile time or run time.

4.4. Is "Code Selection" Traditional Object Oriented Programming? The method to be executed in Julia is not chosen by only one argument, which is what happens in the case of single dispatch, but through multiple dispatch, which considers the types of all the arguments. Julia is not burdened by the encapsulation restrictions (class based methods) of most object oriented languages: The generic functions play a more important role than the data types. Some call this type of language "verb" based as opposed to most object oriented languages being "noun" based. In numerical computing, it is the concept of "solve $A x=b$ " that often seems to be more fundamental, at the highest level, rather than whether the matrix $A$ is full, sparse, or structured. Readers familiar with Java might think, "So what? One can easily create methods based on the types of the arguments." An example is provided in Figure 4. However, a moment's thought shows that the following dynamic situation in Julia is impossible to express in Java:

(Here we use the ternary conditional: if_condition ? value_if_true : value_ if_false.) 


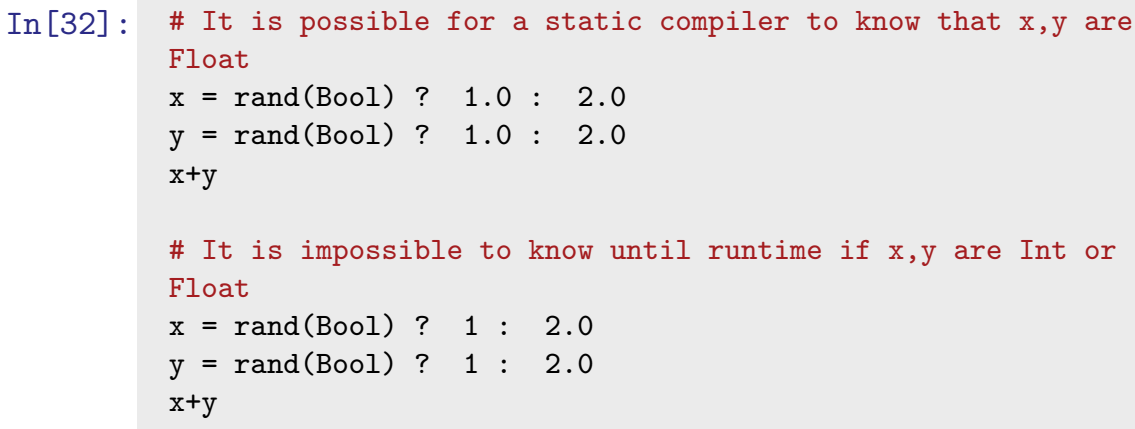

Readers may be familiar with the single dispatch mechanism, as in MATLAB. This implementation is unusual in that it is not completely class based, as the code selection is based on MATLAB's own custom hierarchy. In MATLAB the leftmost object has precedence, but user-defined classes have precedence over built-in classes. MATLAB also has a mechanism to create a custom hierarchy.

Julia generally shuns the notion of "built-in" vs. "user-defined," preferring instead to focus on the method to be performed based on the combination of types and obtaining high performance as a byproduct. A high-level library writer, who we do not distinguish from any other user, has to match the best algorithm to the best input structure. A sparse matrix matches to a sparse routine, a dense matrix to a dense routine. A low-level language designer has to make sure that integers are added with an integer adder, and floating-point numbers are added with a float adder. Despite the very different levels, the reader might recognize that fundamentally, these are both examples of code being selected to match the structure of the problem.

Readers familiar with object oriented paradigms such as $\mathrm{C}++$ or Java are likely familiar with the approach of encapsulating methods inside classes. Julia's more general multiple dispatch mechanism (also known as generic functions, or multi-methods) is a paradigm in which methods are defined on combinations of data types (classes). Julia has proven that this is remarkably well suited for numerical computing. As an aside, in Julia, method ambiguities throw a warning.

A class based language might express the sum of a sparse matrix with a full matrix as follows: A_sparse matrix.plus (A_full matrix). Similarly, it might express indexing as A_sparse_matrix.sub(A_full_matrix). If a tridiagonal were added to the system, one would have to find the method plus or sub which are encapsulated in the sparse matrix class, modify it, and test it. Similarly, one has to modify every full matrix method, etc. We believe that class based methods, which can be taken quite far, are not sufficiently powerful to express the full gamut of abstractions in scientific computing. Furthermore, the burdens of encapsulation create a wall around objects and methods that are counterproductive for numerical computing.

The generic function idea captures the notion that a method for a general operation on pairs of matrices might exist (e.g., "+"), but if a more specific operation is possible (e.g., "+" on sparse matrices or "+" on a special matrix structure like Bidiagonal), then that more specific operation is used. We also mention indexing as another example: why should the indexee take precedence over the index?

4.5. Quantifying the Use of Multiple Dispatch. In [3] we performed an analysis to substantiate the claim that multiple dispatch, an esoteric idea for numerical com- 
Table I A comparison of Julia (1208 functions exported from the Base library) to other languages with multiple dispatch. The "Julia operators" row describes 47 functions with special syntax (binary operators, indexing, and concatenation). Data for other systems are from [26]. The results indicate that Julia is using multiple dispatch far more heavily than previous systems.

\begin{tabular}{|l|r|r|r|}
\hline Language & DR & CR & DoS \\
\hline \hline Gwydion & 1.74 & 18.27 & 2.14 \\
\hline OpenDylan & 2.51 & 43.84 & 1.23 \\
\hline CMUCL & 2.03 & 6.34 & 1.17 \\
\hline SBCL & 2.37 & 26.57 & 1.11 \\
\hline McCLIM & 2.32 & 15.43 & 1.17 \\
\hline Vortex & 2.33 & 63.30 & 1.06 \\
\hline Whirlwind & 2.07 & 31.65 & 0.71 \\
\hline NiceC & 1.36 & 3.46 & 0.33 \\
\hline LocStack & 1.50 & 8.92 & 1.02 \\
\hline Julia & 5.86 & 51.44 & 1.54 \\
\hline Julia operators & 28.13 & 78.06 & 2.01 \\
\hline
\end{tabular}

puting from computer languages, finds its killer application in scientific computing. We wanted to answer for ourselves the question of whether there was really anything different about how Julia uses multiple dispatch.

Table 1 gives an answer in terms of dispatch ratio (DR), choice ratio (CR), and degree of specialization (DoS). While multiple dispatch is an idea that has been circulating for some time, its application to numerical computing appears to have significantly favorable characteristics compared to previous applications.

To quantify how heavily a language feature is used, we use the following metrics $[26]$ :

1. Dispatch ratio: The average number of methods in a generic function.

2. Choice ratio: For each method, the total number of methods over all generic functions it belongs to, averaged over all methods. This is essentially the sum of the squares of the number of methods in each generic function, divided by the total number of methods. The intent of this statistic is to give more weight to functions with a large number of methods.

3. Degree of specialization: The average number of type-specialized arguments per method.

Table 1 shows the mean of each metric over the entire Julia Base library, showing a high degree of multiple dispatch compared with corpora in other languages [26]. Compared to most multiple dispatch systems, Julia functions tend to have a large number of definitions. To see why this might be so, it helps to compare results from a biased sample of common operators. These functions are the most obvious candidates for multiple dispatch, and as a result their statistics climb dramatically. Julia is focused on numerical computing, and so is likely to have a large proportion of functions with this characteristic.

4.6. Case Study for Numerical Computing. The complexity of linear algebra software has been nicely captured in the context of LAPACK and ScaLAPACK by Demmel, Dongarra et al. [7] and is reproduced verbatim here:

(1) for all linear algebra problems

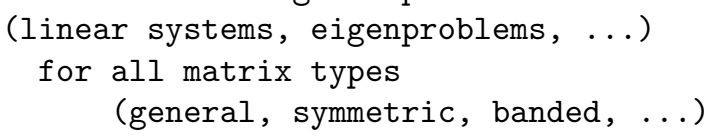




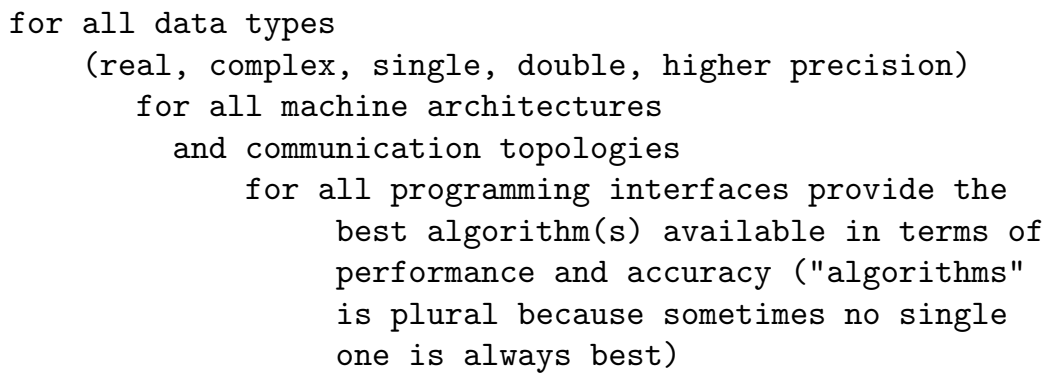

In the language of computer science, code reuse is about taking advantage of polymorphism. In the general language of mathematics it's about taking advantage of abstraction, or the sameness of two things. Either way, programs are efficient, powerful, and maintainable if programmers are given powerful mechanisms to reuse code.

Increasingly, the applicability of linear algebra has gone well beyond the world of floating-point numbers. These days linear algebra is performed on high precision numbers, integers, elements of finite fields, and rational numbers. There will always be a special place for the BLAS and the performance it provides for floating-point numbers. Nevertheless, linear algebra operations transcend any one data type. One must be able to write a general implementation and, as long as the necessary operations are available, the code should just work [27]. That is the power of code reuse.

4.6. I. Determinant: Simple Single Dispatch. In traditional numerical computing there are people with special skills known as library writers. Most users are, well, just users of libraries. In this case study, we show how anybody can dispatch a new determinant function based solely on the type of the argument.

For triangular and diagonal structures, obvious formulas are used. For general matrices, the QR decomposition yields the determinant as the product of the diagonal elements of $R .{ }^{14}$ For symmetric tridiagonals the usual three-term recurrence formula [31] is used. (The first four are defined as one line functions; the symmetric tridiagonal uses the long form.)

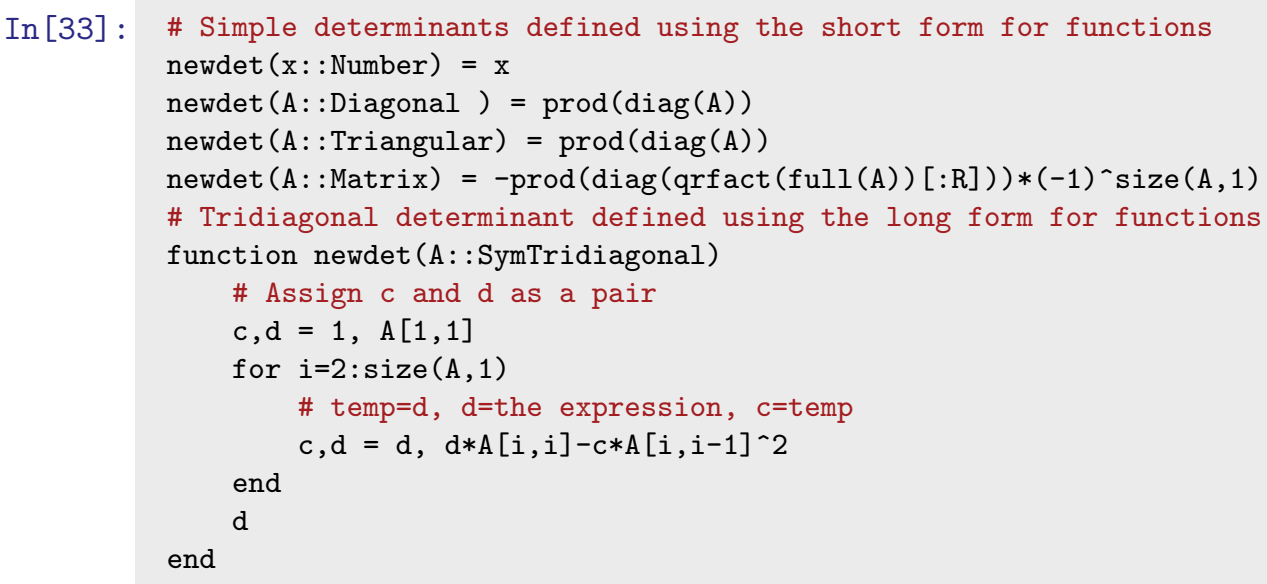

${ }^{14} \mathrm{LU}$ is more efficient. We simply wanted to illustrate that other ways are possible. 
We have illustrated a mechanism to select a formula at run time based on the input type.

4.6.2. A Symmetric Arrow Matrix Type. There exist matrix structures and operations on those matrices. In Julia, these structures exist as Julia types. Julia has a number of predefined matrix structure types: (dense) Matrix, (compressed sparse column) SparseMatrixCSC, Symmetric, Hermitian, SymTridiagonal, Bidiagonal, Tridiagonal, Diagonal, and Triangular are all examples of its matrix structures.

The operations on these matrices exist as Julia functions. Familiar examples of operations are indexing, determinant, size, and matrix addition. Since matrix addition takes two arguments, it may be necessary to reconcile two different types when computing the sum.

In the following Julia example, we illustrate how the user can add symmetric arrow matrices to the system, and then add a specialized det method to compute the determinant of a symmetric arrow matrix efficiently. We build on the symmetric arrow type introduced in section 3.3.

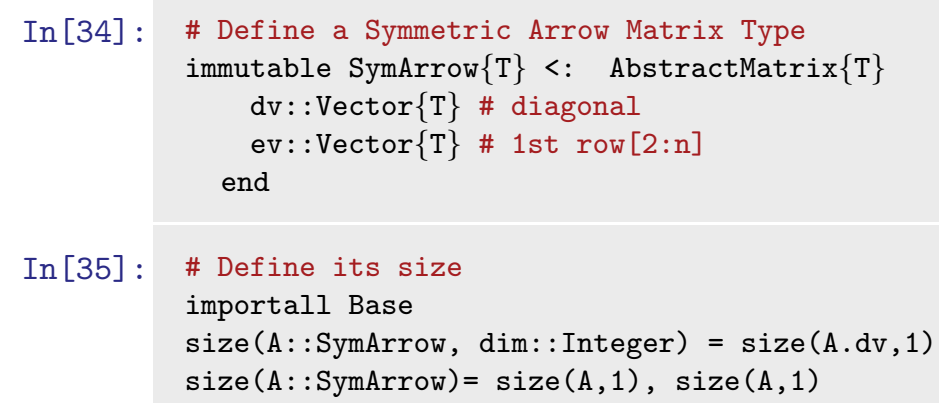

Copyright $@$ by SIAM. Unauthorized reproduction of this article is prohibited. 


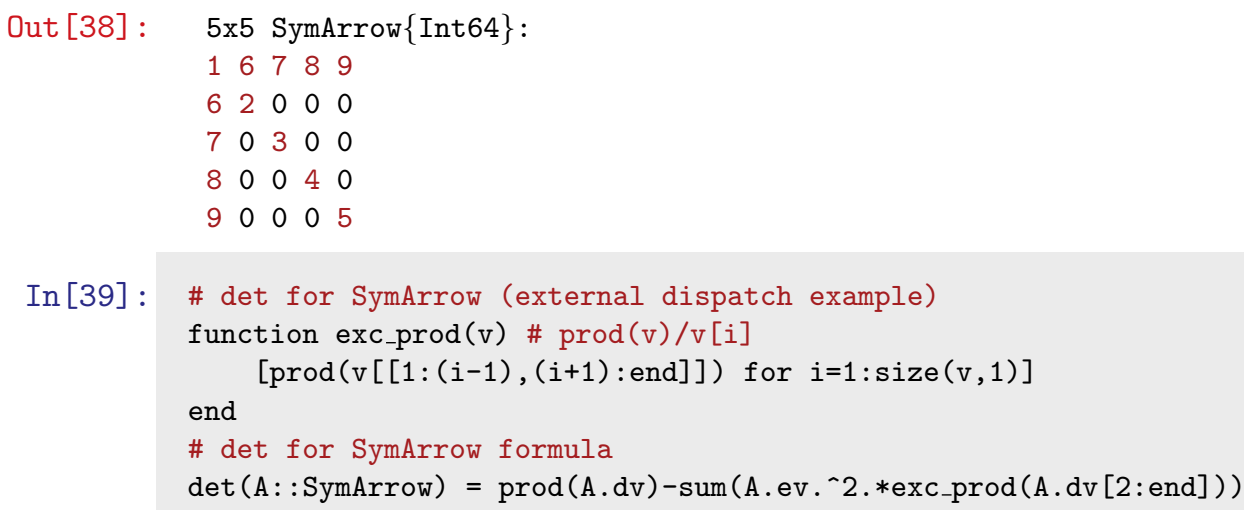

Out [39]: $\operatorname{det}$ (generic function with 17 methods)

The above Julia code uses the formula $\operatorname{det}(A)=\prod_{i=1}^{n} d_{i}-\sum_{i=2}^{n} e_{i}^{2} \prod_{2 \leq j \neq i \leq n} d_{j}$, valid for symmetric arrow matrices where $d$ is the diagonal and $e$ is the first row starting with the second entry.

In some numerical computing languages, a function might begin with a lot of argument checking to pick which algorithm to use. In Julia, one creates a number of methods. Thus, newdet as defined by In [33] on a diagonal is one method for newdet, and newdet on a triangular matrix is a second method. In practice, one would overload det itself as shown in In [39] for SymArrow matrices: det on a SymArrow is a new method for det. (See section 4.6.1.)

We have now seen a number of examples of code selection for single dispatch, i.e., the selection of code based on the type of a single argument. We might notice that a symmetric arrow plus a diagonal does not require operations on full dense matrices. The code below starts with the most general case, and then allows for specialization for the symmetric arrow and diagonal sum:

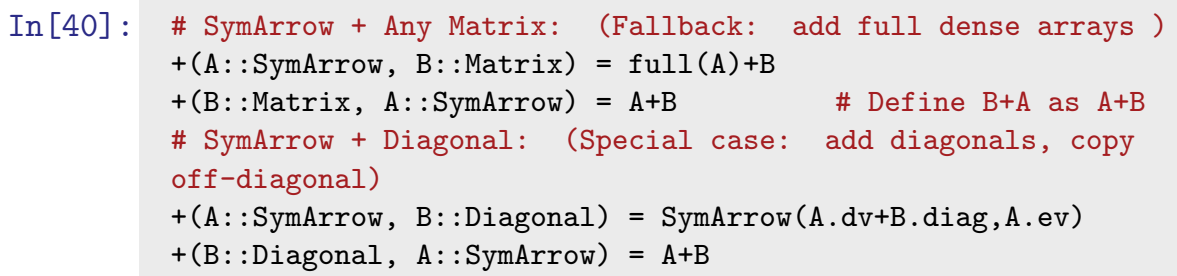

5. Leveraging Design for High Performance Libraries. Seemingly innocuous design choices in a language can have profound, pervasive performance implications. These are often overlooked in languages that were not designed from the beginning to be able to deliver excellent performance. See Figure 5. Other aspects of language and library design affect the usability, composability, and power of the provided functionality.

5.I. Integer Arithmetic. A simple but crucial example of a performance-critical language design choice is integer arithmetic. Consider what happens if we make a fixed 


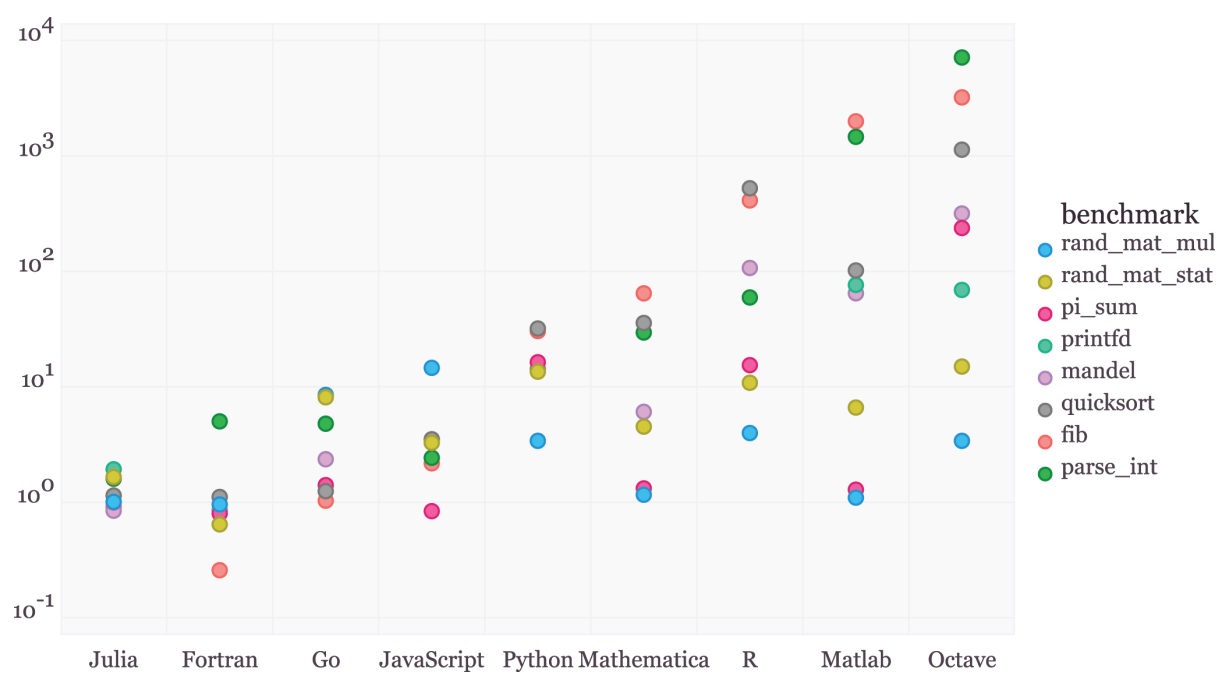

Fig. 5 Performance comparison of various languages performing simple microbenchmarks. Benchmark execution time relative to $C$. (Smaller is better; $C$ performance =1.0.)

number of loop iterations on an integer argument:

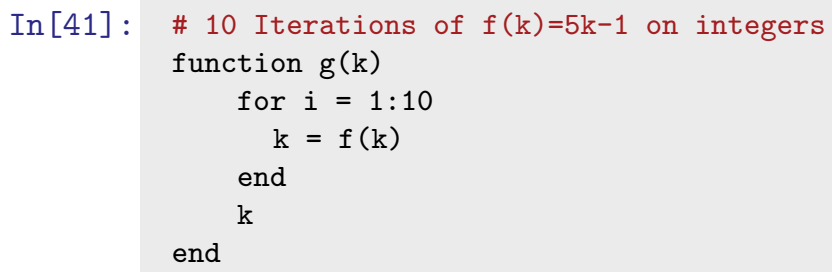

Because the compiler knows that integer addition and multiplication are associative and that multiplication distributes over addition, it can optimize the entire loop down to just a multiply and an add. Indeed, if $f(k)=5 k-1$, it is true that the tenfold iterate $f^{(10)}(k)=-2441406+9765625 k$.

\subsection{A Powerful Approach to Linear Algebra.}

5.2.I. Matrix Factorizations. We describe how Julia's features have been used to provide a powerful approach to linear algebra [27]. For decades, orthogonal matrices have been represented internally as products of Householder matrices displayed for humans as matrix elements. $L U$ factorizations are often performed in place, storing the $L$ and $U$ information together in the data locations originally occupied by $A$. All this speaks to the fact that matrix factorizations deserve a natural place in a linear algebra library.

In Julia, thanks to the contributions of Andreas Noack [27] and many others, these structures are indeed first class objects. The structure QRCompactWYQ holds a 
compact $Q$ and an $R$ in memory. Similarly, an LU holds an $L$ and a $U$ in packed form in memory. Through the magic of multiple dispatch, we can solve linear systems, extract the pieces, and do least squares directly on these structures.

The $Q R$ example is even more fascinating. Suppose one computes $Q R$ of a $4 \times 3$ matrix. What is the size of $Q$ ? The right answer, of course, is that it depends: it could be $4 \times 4$ or $4 \times 3$. The underlying representation is the same: It is the product of three Householder matrices.

In Julia one can compute Aqr $=\operatorname{qrfact}(\operatorname{rand}(4,3))$, then one can extract $Q$ from the factorization with $\mathrm{Q}=\operatorname{Aqr}[: \mathrm{Q}]$. (Note that :Q is a symbol; the syntax Aqr [:Q] is a shorthand for Base.LinAlg.getq(Aqr).)

This $Q$ retains its clever underlying structure and is therefore efficient and applicable when multiplying vectors of length 4 or length 3, contrary to the rules of freshman linear algebra, but welcome in numerical libraries for saving space and enabling faster computations.

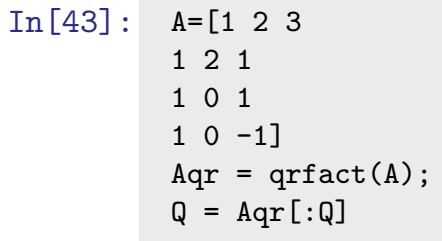

5.2.2. User-Extensible Wrappers for BLAS and LAPACK. The tradition is to leave the coding to LAPACK writers and call LAPACK for speed and accuracy. This has worked fairly well, but Julia exposes considerable opportunities for improvement.

Julia users have access to a variety of linear algebra operations available directly from Julia without needing to know anything about LAPACK. All of LAPACK is available, not just the most common functions. LAPACK wrappers are implemented 
fully in Julia code, using ccall, ${ }^{15}$ which does not require a $\mathrm{C}$ compiler and can be called directly from the interactive Julia prompt.

Consider the Cholesky factorization by calling LAPACK's XPOTRF. It uses Julia's metaprogramming facilities to generate four functions, corresponding to the XPOTRF functions for Float32, Float64, Complex64, and Complex128 types. The call to the Fortran functions is wrapped in ccall.

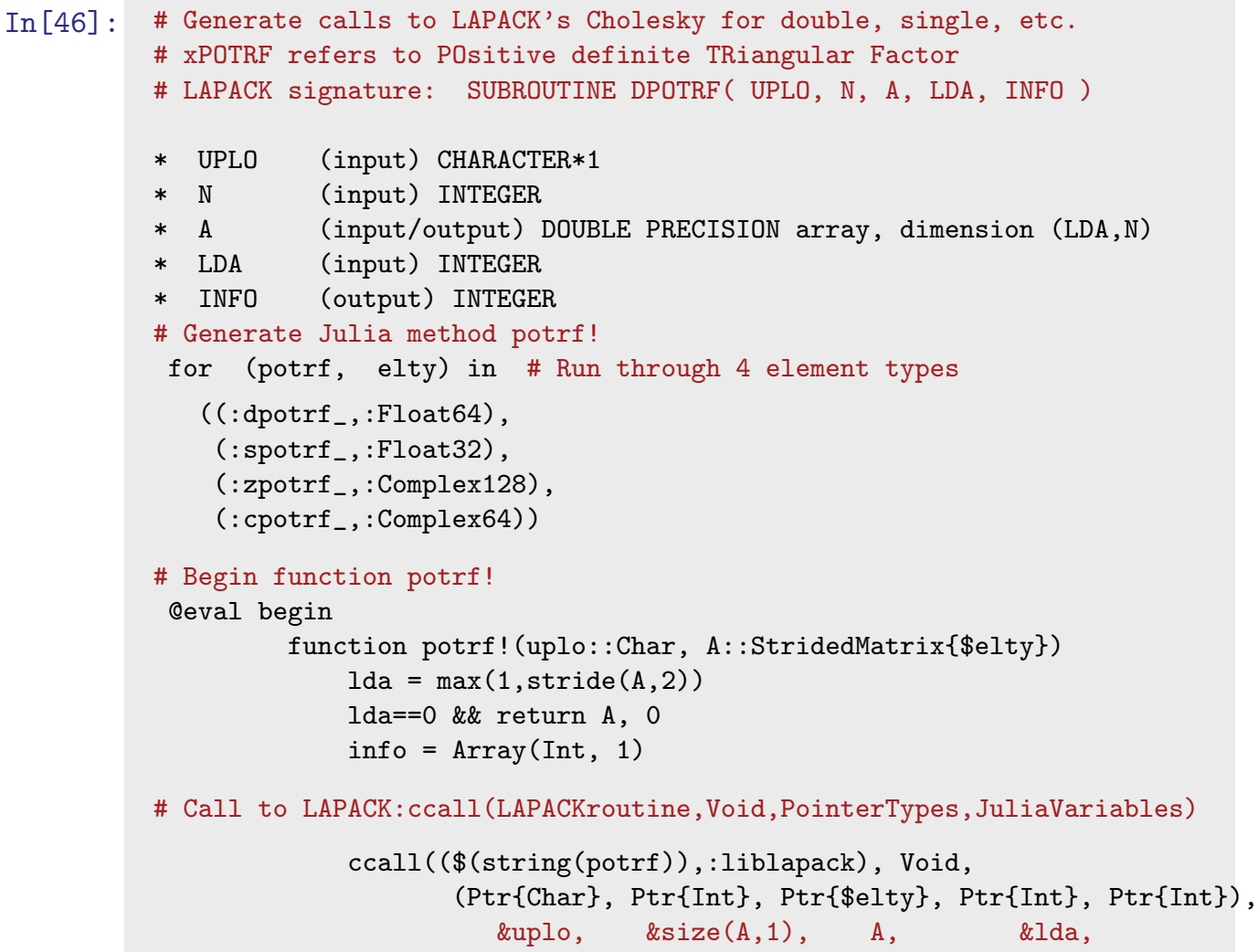

5.3. High Performance Polynomials and Special Functions with Macros. Julia has a macro system that provides custom code generation, providing performance that is otherwise difficult to achieve. A macro is a function that runs at parse time, takes symbolic expressions in, and returns transformed expressions out, which are inserted into the code for later compilation. For example, a library developer has implemented an @evalpoly macro that uses Horner's rule to evaluate polynomials efficiently. Consider

In [47]: Qevalpoly $(10,3,4,5,6)$

\footnotetext{
${ }^{15} \mathrm{http}$ ///docs.julialang.org/en/latest/manual/calling-c-and-fortran-code/
} 
which returns 6543 (the polynomial $3+4 x+5 x^{2}+6 x^{3}$, evaluated at 10 with Horner's rule). Julia allows us to see the inline generated code with the command

In [48]: macroexpand (: @evalpoly $(10,3,4,5,6))$

We reproduce the key lines below

Out [48]: \#471\#t $=10$ \# Store 10 into a variable named \#471\#t

Base.Math.+(3, Base.Math.*(\#471\#t, Base.Math.+(4, Base.Math.*

$(\# 471 \#$, Base.Math.+(5,Base.Math.*(\#471\#t,6)))) ))

This code-generating macro only needs to produce the correct symbolic structure, and Julia's compiler handles the remaining details of fast native code generation. Since polynomial evaluation is so important for numerical library software it is critical that users can evaluate polynomials as fast as possible. The overhead of implementing an explicit loop, accessing coefficients in an array, and possibly a subroutine call (if it is not inlined) is substantial compared to just inlining the whole polynomial evaluation.

The polynomial macro may be expanded to work on a matrix first argument by defining Base. muladd $(x, y, z)=x * y+z * I$.

5.4. Easy and Flexible Parallelism. Parallel computing remains an important research topic in numerical computing, and has yet to reach the level of richness and interactivity required. The issues discussed in section 3.1 on the balance between the human and the computer become more pronounced in the parallel setting. Part of the problem is that parallel computing means different things to different people:

1. At the most basic level, one wants instruction-level parallelism within a CPU and expects the compiler to discover such parallelism. In Julia, this can be achieved with the @simd primitive.

2. In order to utilize multicore and manycore CPUs on the same node, one wants multithreading. Currently, we have experimental multithreading support in Julia, and this will be the topic of a further paper. Julia currently does provide a SharedArray data structure where the same array in memory can be operated on by multiple different Julia processes on the same node.

3. Distributed memory is often considered to be the most difficult model. This can mean running Julia on anything from half a dozen and thousands of nodes, each with multicore CPUs.

Our experience with Star-P [5] taught us valuable lessons. Star-P parallelism $[14,13]$ includes global dense, sparse, and cell arrays that are distributed on parallel shared or distributed memory computers. Before the evolution of the cloud as we know it today, the user used a familiar frontend (e.g., MATLAB) as the client on a laptop or desktop and connected seamlessly to a server (usually a large distributed computer). Blockbuster functions from sparse and dense linear algebra, parallel FFTs, parallel sorting, and many others, were easily available and composable for the user. In these cases Star-P called Fortran/MPI or C/MPI. Star-P also allows a kind of parallel for loop that works on rows, planes, or hyperplanes of an array. In these cases Star-P uses copies of the client language on the backend, usually MATLAB, Octave, Python, or R.

We learned that while we were able to obtain a useful parallel system in this way, bolting parallelism onto an existing language that was not designed for performance is difficult at best, and impossible at worst. One of our motivations to build Julia was to design the right language for parallel computing.

Copyright (c) by SIAM. Unauthorized reproduction of this article is prohibited. 
Julia provides many facilities for parallelism, which are described in detail in the Julia manual. ${ }^{16}$ Distributed memory programming in Julia is built on two primitivesremote calls that execute a function on a remote processor and remote references that are returned by the remote processor to the caller. These primitives are implemented completely within Julia. On top of them, Julia provides a distributed array data structure, a pmap implementation, and a way to parallelize independent iterations of a loop with the @parallel macro, all of which can parallelize code in distributed memory. These ideas are exploratory in nature, and we only discuss them here to emphasize that well-designed programming language abstractions and primitives allow one to express and implement parallelism completely within the language.

We proceed with one example that demonstrates parallel computing at work and shows how one can impulsively grab a large number of processors and explore their problem space quickly.

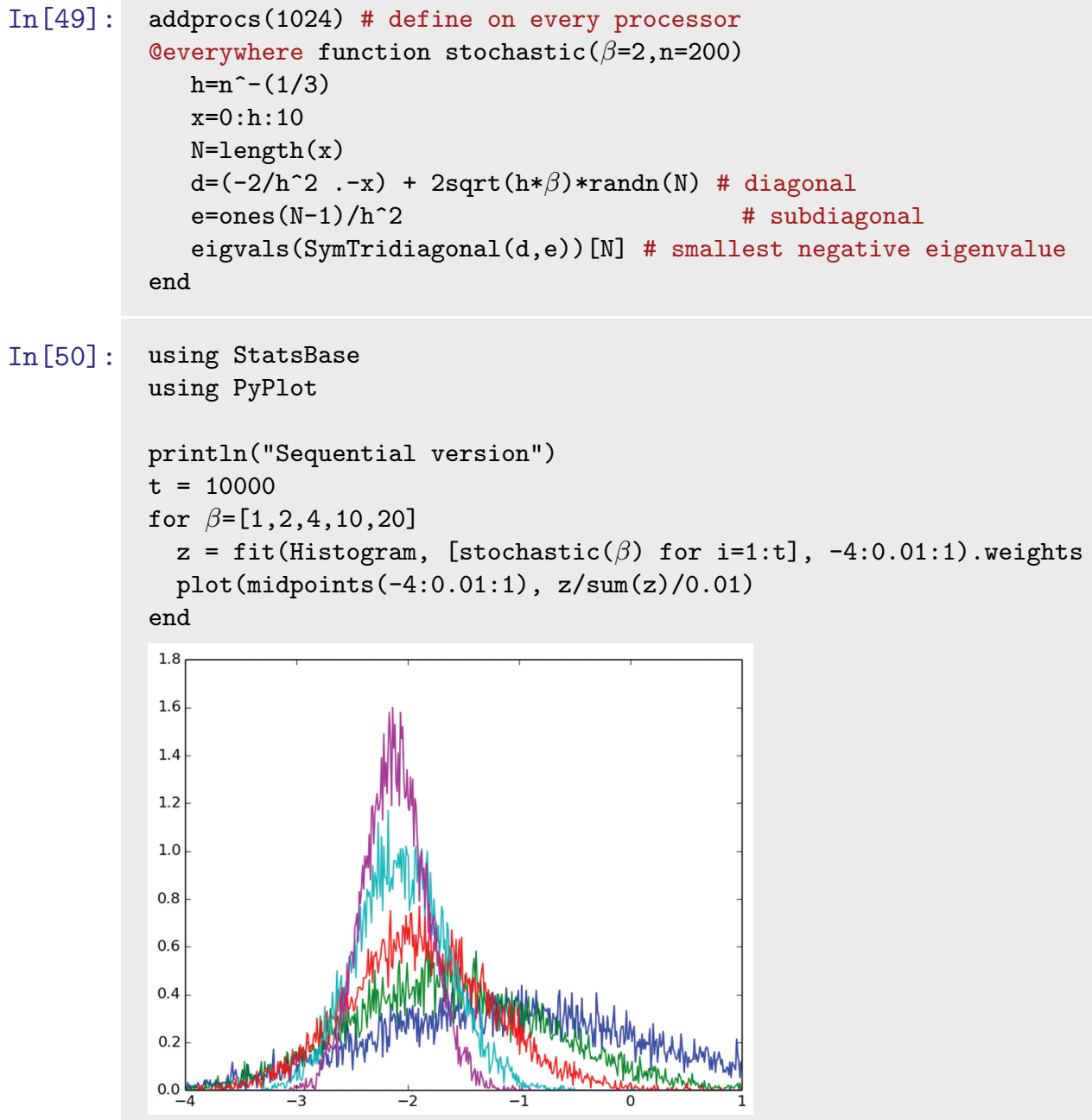

${ }^{16} \mathrm{http}: / /$ docs.julialang.org/en/latest/manual/parallel-computing/ 
Suppose we wish to perform a complicated histogram in parallel. We use an example from random matrix theory, (but it could easily have been from finance), the computation of the scaled largest eigenvalue in magnitude of the so-called stochastic Airy operator: [8] $\frac{d^{2}}{d x^{2}}-x+\frac{1}{2 \sqrt{\beta}} d W$. This is the usual finite difference discretization of $\frac{d^{2}}{d x^{2}}-x$ with a "noisy" diagonal.

We illustrate an example of the famous Tracy-Widom law being simulated with Monte Carlo experiments for different values of the inverse temperature parameter $\beta$. The simulation on one processor is fuzzy and unfocused, as compared to the same simulation on 1024 processors, which is sharp and focused and runs in exactly the same wall clock time as the sequential run.

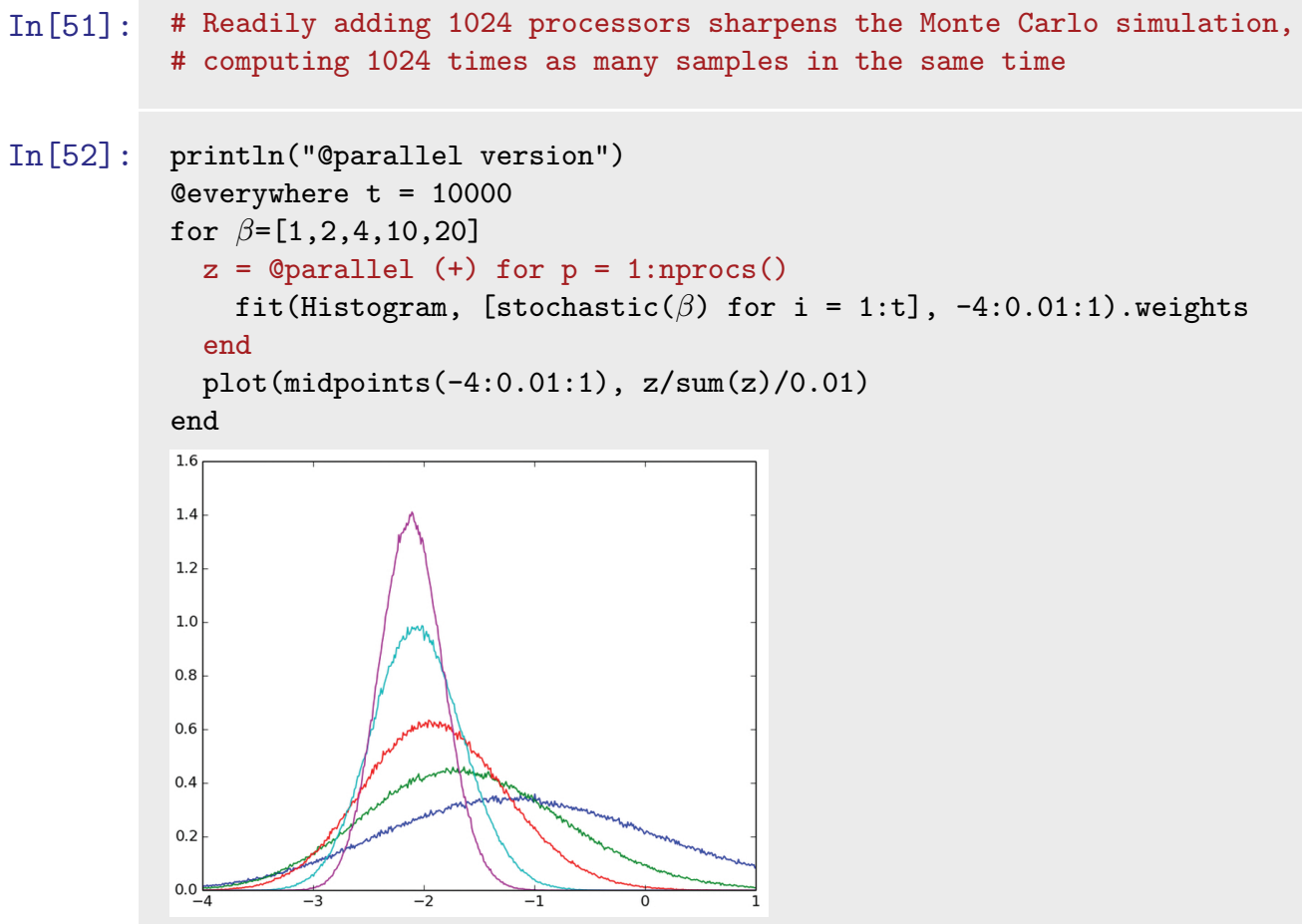

5.5. Performance Recap. In the early days of high-level numerical computing languages, the thinking was that the performance of the high-level language did not matter so long as most of the time was spent inside the numerical libraries. These libraries consisted of blockbuster algorithms that would be highly tuned, making efficient use of computer memory, cache, and low-level instructions.

What the world learned was that only a few codes spent a majority of their time in the blockbusters. Most codes were being hampered by interpreter overheads, stemming from processing more aspects of a program at run time than are strictly necessary.

As we explored in section 3 , one of the hindrances to completing this analysis is type information. Programming language design thus becomes an exercise in balancing incentives to the programmer to provide type information and the ability of the computer to infer type information. Vectorization is one such incentive system. 
Existing numerical computing languages would have us believe that this is the only system or, even if there are others, that somehow this is the best system.

Vectorization at the software level can be elegant for some problems. There are many matrix computation problems that look beautiful vectorized. These programs should be vectorized. Other programs require heroics and skill to vectorize, sometimes producing unreadable code all in the name of performance. These programs we object to vectorizing. Still other programs cannot be vectorized very well, even with heroics. The Julia message is to vectorize when it is natural, producing nice code. Do not vectorize in the name of speed.

Some users believe that vectorization is required to make use of special hardware capabilities such as SIMD instructions, multithreading, GPU units, and other forms of parallelism. This is not strictly true, as compilers are increasingly able to apply these performance features to explicit loops. The Julia message remains the same: vectorize when natural, when you feel it is right.

6. Conclusion. We built Julia to meet our needs for numerical computing, and it turns out that many others wanted exactly the same thing. At the time of writing, not a day goes by when we don't learn that someone new has picked up Julia at universities and companies around the world, in fields as diverse as engineering, mathematics, physical and social sciences, finance, biotech, and many others. More than just a language, Julia has become a place for programmers, physical scientists, social scientists, computational scientists, mathematicians, and others to pool their collective knowledge in the form of online discussions and code.

Acknowledgments. Julia would not have been possible without the enthusiasm and contributions of the Julia community ${ }^{17}$ and of MIT during early years of its development. We thank Michael La Croix for his beautiful Julia display macros. We are indebted at MIT to Jeremy Kepner, Chris Hill, Saman Amarasinghe, Charles Leiserson, Steven Johnson, and Gil Strang for their collegial support, which not only allowed for the possibility of an academic research project to update technical computing, but made it more fun, too.

\section{REFERENCES}

[1] E. Anderson, Z. Bai, C. Bischof, S. Blackford, J. Demmel, J. Dongarra, J. Du Croz, A. Greenbaum, S. Hammarling, A. McKenney, and D. Sorensen, LAPACK Users' Guide, 3rd ed., SIAM, Philadelphia, PA, 1999. (Cited on p. 73)

[2] J. Bezanson, Abstraction in Technical Computing, Ph.D. thesis, Massachusetts Institute of Technology, MA, 2015. (Cited on p. 68)

[3] J. Bezanson, J. Chen, S. Karpinski, V. B. Shah, and A. Edelman, Array operators using multiple dispatch, in ARRAY'14: Proceedings of ACM SIGPLAN International Workshop on Libraries, Languages, and Compilers for Array Programming, ACM, New York, 2014, pp. 56:56-56:61. (Cited on p. 86)

[4] J. Bezanson, S. Karpinski, V. B. Shah, and A. Edelman, Julia: A Fast Dynamic Language for Technical Computing, preprint, arXiv:1209.5145 [cs.PL], 2012. (Cited on pp. 68, 78)

[5] R. Choy and A. Edelman, Parallel MATLAB: Doing it right, Proc. IEEE, 93 (2005), pp. 331341. (Cited on p. 94)

[6] Clang: A C Language Family Frontend for LLVM, http://clang.llvm.org/. (Cited on p. 68)

[7] J. W. Demmel, J. J. Dongarra, B. N. Parlett, W. Kahan, M. Gu, D. S. Bindel, Y. Hida, X. S. Li, O. A. Marques, E. J. Riedy, C. Vomel, J. Langou, P. Luszczek, J. Kurzak, A. Buttari, J. Langou, and S. Tomov, Prospectus for the Next LAPACK and ScaLAPACK Libraries, Tech. report 181, LAPACK Working Note, 2007, http://www.netlib.org/lapack/lawnspdf/lawn181.pdf. (Cited on p. 87)

\footnotetext{
${ }^{17}$ https://github.com/JuliaLang/julia/graphs/contributors
} 
[8] A. Edelman and B. Sutton, From Random Matrices to Stochastic Operators, J. Statist. Phys., 127 (2007), pp. 1121-1165. (Cited on p. 96)

[9] The GNU MPFR Library, http://www.mpfr.org/. (Cited on p. 74)

[10] C. Gomez, ED., Engineering and Scientific Computing with Scilab, Birkhäuser, Boston, 1999. (Cited on p. 66)

[11] G. HoARe, Technicalities: Interactive Scientific Computing \#1 of 2: Pythonic Parts, http: //graydon2.dreamwidth.org/3186.html, 2014. (Cited on p. 74)

[12] R. Ihaka And R. Gentleman, $R$ : A language for data analysis and graphics, J. Comput. Graph. Statist., 5 (1996), pp. 299-314. (Cited on p. 66)

[13] Interactive Supercomputing, Star-p user guide. http://www-math.mit.edu/ edelman/ publications/star-p-user.pdf. (Cited on p. 94)

[14] Interactive Supercomputing, Getting Started with Star-P: Taking Your First Test-Drive, http://www-math.mit.edu/ edelman/publications.php, 2006. (Cited on p. 94)

[15] The Jupyter Project, http://jupyter.org/. (Cited on p. 67)

[16] W. Kahan, How Futile Are Mindless Assessments of Roundoff in Floating-Point Computation?, http://www.cs.berkeley.edu/ $\sim_{\text {wkahan/Mindless.pdf, 2006. (Cited on p. } 72 \text { ) }}$

[17] M. A. Kaplan and J. D. Ullman, A scheme for the automatic inference of variable types, J. ACM, 27 (1980), pp. 128-145, https://doi.org/10.1145/322169.322181. (Cited on p. 78)

[18] C. LatTner And V. AdVE, LLVM: A compilation framework for lifelong program analysis and transformation, in Proceedings of the 2004 International Symposium on Code Generation and Optimization (CGO'04), Palo Alto, CA, 2004, ACM, New York, 2004, pp. 75-86. (Cited on p. 68)

[19] C. L. Lawson, R. J. Hanson, D. R. Kincaid, and F. T. Krogh, Basic linear algebra subprograms for Fortran usage, ACM Trans. Math. Softw., 5 (1979), pp. 308-323, https://doi.org/10.1145/355841.355847. (Cited on p. 73)

[20] M. Lubin And I. Dunning, Computing in Operations Research using Julia, INFORMS J. Comput., 27 (2015), pp. 238-248, https://doi.org/10.1287/ijoc.2014.0623; arXiv preprint: http://dx.doi.org/10.1287/ijoc.2014.0623. (Cited on pp. 67, 74)

[21] Mathematica, http://www.mathematica.com. (Cited on p. 66)

[22] MATLAB, http://www.mathworks.com. (Cited on p. 66)

[23] The MIT License, http://opensource.org/licenses/MIT. (Cited on p. 74)

[24] M. Mohnen, A graph-free approach to data-flow analysis, in Compiler Construction, R. Horspool, ed., Lecture Notes in Comput. Sci. 2304, Springer, Berlin, Heidelberg, 2002, pp. 185213. (Cited on p. 78)

[25] M. Murphy, Octave: A free, high-level language for mathematics, Linux J., 1997 (1997), 326884, http://dl.acm.org/citation.cfm?id=326876.326884. (Cited on p. 66)

[26] R. Muschevici, A. Potanin, E. Tempero, and J. Noble, Multiple dispatch in practice, in Proceedings of the 23rd ACM SIGPLAN Conference on Object-Oriented Programming Systems Languages and Applications, OOPSLA '08, ACM, New York, 2008, pp. 563-582, https://doi.org/10.1145/1449764.1449808. (Cited on p. 87)

[27] A. NoACK, Fast and Generic Linear Algebra in Julia, Tech. report, MIT, Cambridge, MA, 2015. (Cited on pp. 88, 91)

[28] J. Regier, K. Pamnany, R. Giordano, R. Thomas, D. Schlegel, J. McAuliffe, and PrabHAT, Learning an Astronomical Catalog of the Visible Universe through Scalable Bayesian Inference, preprint, arXiv:1611.03404 [cs.DC], 2016. (Cited on p. 67)

[29] Rust, http://www.rust-lang.org/. (Cited on p. 74)

[30] H. Shen, Interactive notebooks: Sharing the code, Nature Toolbox, 515 (2014), pp. 151-152, http://www.nature.com/news/interactive-notebooks-sharing-the-code-1.16261. (Cited on p. 67)

[31] G. Strang, Introduction to Linear Algebra, Wellesley-Cambridge Press, Wellesley, MA, 2003, https://books.google.com/books?id=Gv4pCVyoUVYC. (Cited on p. 88)

[32] Swift, https://developer.apple.com/swift/. (Cited on p. 68)

[33] M. Udell, K. Mohan, D. Zeng, J. Hong, S. Diamond, and S. Boyd, Convex optimization in Julia, in SC14 Workshop on High Performance Technical Computing in Dynamic Languages, 2014; preprint, arXiv:1410.4821 [math.OC], 2014. (Cited on p. 67)

[34] S. van der Walt, S. C. Colbert, and G. Varoquaux, The NumPy Array: A Structure for Efficient Numerical Computation, CoRR, abs/1102.1523, 2011. (Cited on p. 66)

[35] H. WiCKHAM, ggplot2, http://ggplot2.org/. (Cited on p. 71)

[36] L. Wilkinson, The Grammar of Graphics (Statistics and Computing), Springer-Verlag, New York, 2005. (Cited on p. 71)

Copyright $@$ by SIAM. Unauthorized reproduction of this article is prohibited. 